\title{
CT-Guided Percutaneous Biopsy of Intrathoracic Lesions
}

\author{
Hira Lal, MD ${ }^{1}$, Zafar Neyaz, MD ${ }^{1}$, Alok Nath, MD, DM², Samudra Borah, MD ${ }^{1}$ \\ Departments of ${ }^{1}$ Radiodiagnosis and ${ }^{2}$ Pulmonary Medicine, Sanjay Gandhi Post Graduate Institute of Medical Sciences, Lucknow, Uttar Pradesh \\ 226014, India
}

Percutaneous CT-guided needle biopsy of mediastinal and pulmonary lesions is a minimally invasive approach for obtaining tissue for histopathological examination. Although it is a widely accepted procedure with relatively few complications, precise planning and detailed knowledge of various aspects of the biopsy procedure is mandatory to avert complications. In this pictorial review, we reviewed important anatomical approaches, technical aspects of the procedure, and its associated complications. Index terms: Biopsy; Computed tomography; Interventional radiology; Lung; Mediastinum; Pneumothorax

\section{INTRODUCTION}

Percutaneous CT-guided needle biopsy (PCNB) of the intrathoracic lesions is a well-established technique for obtaining tissue for histopathological examination and various other tests $(1,2)$. Although this technique is less invasive as compared to the surgical biopsy, complications sometimes do occur. Due to the presence of the heart, major vessels, bones, and the lung parenchyma, the operator has to be well versed with the various anatomical and technical aspects of the biopsy procedure in order to minimize complications. In this article, we have tried to present various aspects of percutaneous CT-guided needle biopsy of mediastinal and lung lesions.

Received August 17, 2011; accepted after revision October 4, 2011. Corresponding author: Zafar Neyaz, MD, Department of Radiodiagnosis, Sanjay Gandhi Post Graduate Institute of Medical Sciences, Rae Bareilly Road, Lucknow, Uttar Pradesh 226014, India.

- Tel: (91) 0522-2668700 Extn.4590 - Fax: (91) 0522-2668017

- E-mail: zafarneyaz@hotmail.com

This is an Open Access article distributed under the terms of the Creative Commons Attribution Non-Commercial License (http://creativecommons.org/licenses/by-nc/3.0) which permits unrestricted non-commercial use, distribution, and reproduction in any medium, provided the original work is properly cited.

\section{Prebiopsy Preparation}

Several steps are taken in the preparation of each biopsy procedure $(2,3)$. Informed consent is obtained from the patient and the risks, benefits, and alternatives are discussed in detail. Any anticoagulant medication is discontinued at least 4-5 days before the procedure (2, 4). Although there have been no definite guidelines for PCNB in patients using an anti-platelet medication, some authors have recommended discontinuing aspirin at least 5 days before a biopsy is performed (3). A recent platelet count and complete coagulogram should be available before the procedure to exclude any coagulation abnormality. A good quality baseline contrast-enhanced CT should always be done and reviewed before the procedure for the lesion size, location, lesion vascularity, and important structures located in the biopsy path. The condition of the underlying lung is evaluated for the presence of emphysema and bullous lesions. A violent uncooperative patient is one of the major contraindications for PCNB. Other relative contraindications include bleeding diatheses, severe bullous emphysema, contralateral pneumonectomy, hydatid cyst, pulmonary hypertension and a highly vascular lesion (Fig. 1) $(3,4)$. If the patient has cough, cough suppressants should be prescribed. 

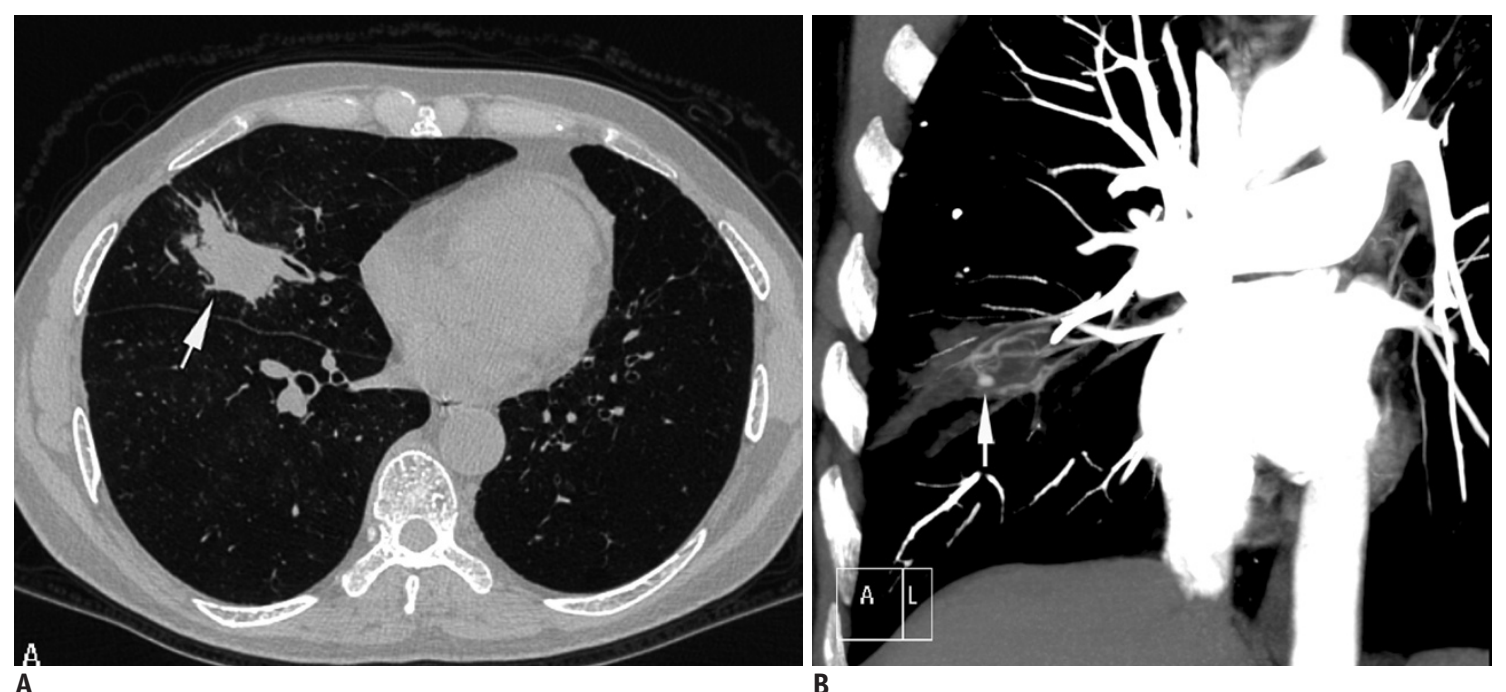

Fig. 1. Fifty-six-year-old man who had previously received antitubercular treatment, presented with recurrent episodes of hemoptysis and low grade fever.

A. Axial lung window CT image showed nodular lesion in right middle lobe with irregular margins (arrow). B. Coronal maximum-intensityprojection imaging after contrast injection shows multiple hypertrophied arteries and aneurysm (arrow) inside lesion. As result of these findings, biopsy was not attempted. Patient responded to empiric antitubercular treatment and final diagnosis of reactivation tuberculosis was made.

\section{Biopsy Procedure and Various Techniques for Lesion Targeting}

\section{Medication and Monitoring}

Patent wide bore (18-20 G) intravenous access should be obtained and a facility for continuous pulse oximetry and vital monitoring should be available. Sedation is generally not desirable as it reduces the patient's level of cooperation. However, whenever sedation is necessary, 0.25 $\mathrm{mg}$ of alprazolam can be given orally or as an alternative, $1 \mathrm{mg}$ of midazolam can be administered intravenously (4). The procedure is done under local anesthesia (1-2\% lignocaine). While injecting the local anesthetic, care is taken not to advance needle tip into pleura or any vascular structure.

\section{Choosing the Appropriate Biopsy Needle}

Percutaneous biopsies consist of mainly two types: fine needle aspiration biopsy and cutting or core biopsy $(2,3)$. Fine needle aspiration biopsy is also known as fine needle aspiration cytology or fine needle aspiration. Aspiration needles are usually 20-25 gauge and provide material for cytological and microbiological examination (Fig. 2). Cutting or core biopsy needles provide small linear tissue sections suitable for histological evaluation. These needles are usually larger in caliber than aspiration needles; however, nowadays small caliber (18-20 gauge) automated cutting needles are available. The diagnostic accuracy of aspiration

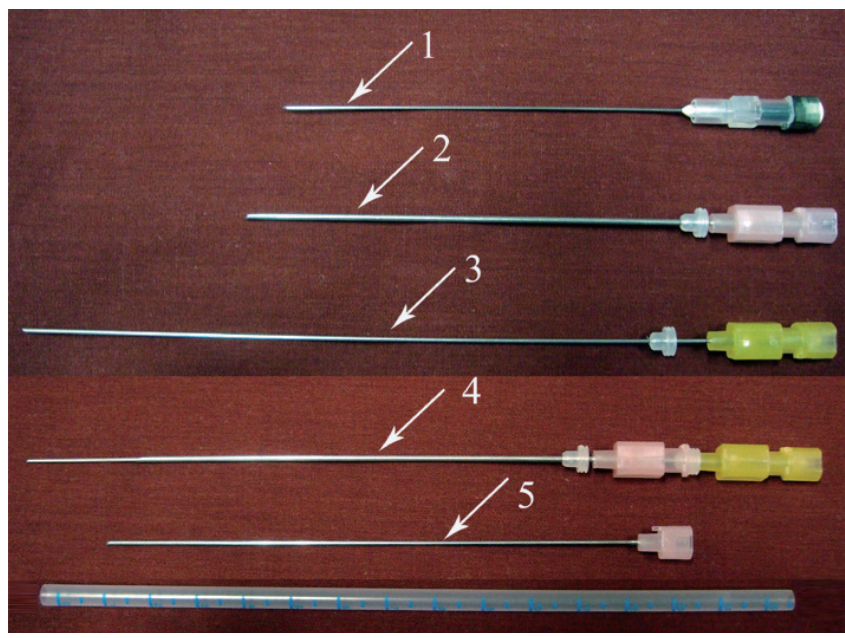

Fig. 2. Some commonly used aspiration needles. 1, 22-gauge $89 \mathrm{~mm}$ long spinal needle; 2, 18-gauge $10 \mathrm{~cm}$ long chiba needle; 3, 20-gauge $15 \mathrm{~cm}$ long chiba needle; 4, Coaxial system had been formed by placing 20 -gauge needle into 18-gauge needle; 5 , Stylet of 18-gauge chiba needle.

biopsy is almost as good as core biopsy for the diagnosis of malignant lesions, especially if an onsite cytopathologist is present. However, for the diagnosis of benign lesions and lymphoma, core biopsy is preferred $(2,3,5)$. Although rates for pneumothorax are similar for aspiration needles and cutting biopsy needles, a slightly higher incidence of pulmonary bleeding is reported with cutting biopsy needles (2). Moreover, there is little evidence in the literature that needle gauge affects the complication rate within the size range of the smaller needles available for lung 
biopsy; needles larger than 18-gauge are considered a risk for causing both bleeding and pneumothorax (6). During the biopsy of mediastinal lesions, large-caliber needles should be avoided if the needle path traverses the lung parenchyma, when great vessels are located in proximity to the lesion, or if the lesion is highly vascular (5).

Both aspiration and core biopsies can be done either by using a single needle technique or a coaxial technique. In the single needle technique, a needle is directly advanced into the lesion and if multiple samples are required each time, a new pass will be made. Alternatively, a coaxial technique can be used, and involves the initial placement of an outer guiding needle close to the target, followed by the introduction of a thinner biopsy needle through it to sample the lesion (6). The disadvantage of the single needle technique is that each time the needle is introduced into the lesion; image guidance is required, resulting in increased procedure time. Moreover, intervening structures are traversed each time, resulting in increased risk of complication (5). While a coaxial technique offers many advantages, larger caliber guiding needles are required to puncture the pleura. In the presence of a prominent internal air-bronchogram or open-bronchus sign in the lesion, a coaxial technique should be used more carefully as there is an increased risk of air embolism.

To employ the coaxial technique during aspiration biopsy, an 18-gauge chiba needle can be used as the guide, followed by insertion of a longer length 20-22 gauge chiba needle (Fig. 2) (5). In our institute, we use the coaxial biopsy set containing a 16 or 19 -gauge guiding needle with an 18 or 20-gauge biopsy needle, respectively for obtaining tissue cores (Quick core biopsy set, Cook, Bloomington, IN, USA) (Fig. 3). Needle length should be selected depending on depth of lesion from the skin. In the quick core biopsy set available at our institution the outer guiding needle is 3-4 cm shorter than the inner biopsy needle and this is a very important point to be remembered during the needle selection. A quick core biopsy needle is available with two different lengths of specimen notch, $10 \mathrm{~mm}$ and $20 \mathrm{~mm}$. A longer specimen notch is preferred as it gives more tissue; however, if the lesion is small, a short notch is used to avoid injury to the normal structures. One more advantage of quick core needle is that the specimen notch can be advanced into the lesion and the exact site of the biopsy can be ascertained prior to firing the biopsy needle.

After reviewing the previous images, a safe route to the lesion and an appropriate sized needle is chosen. Needle

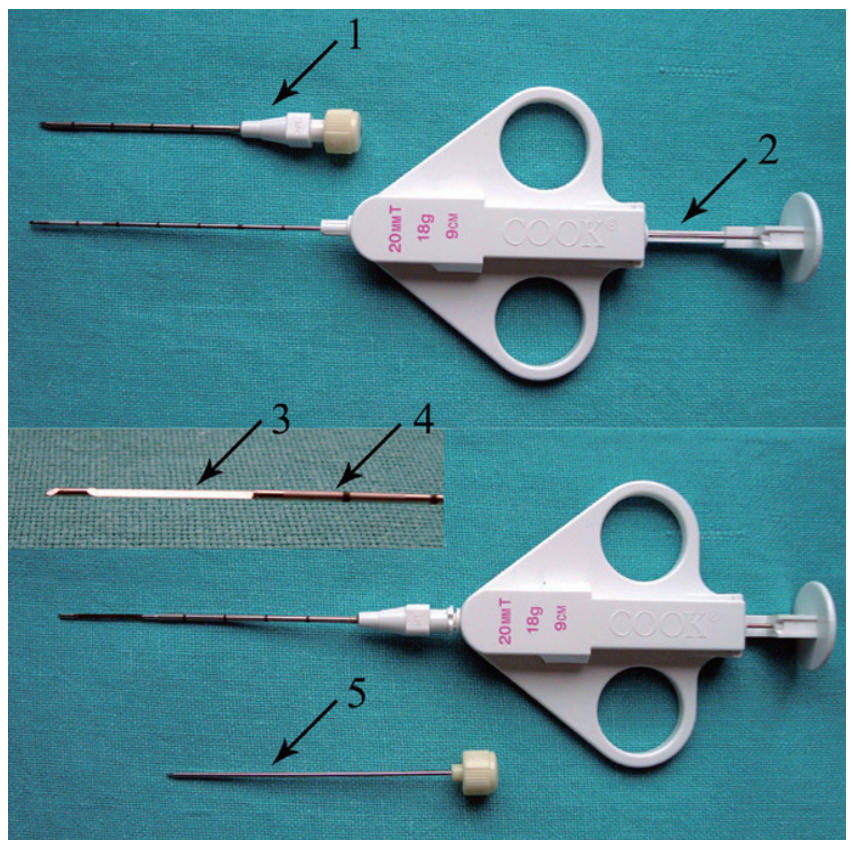

Fig. 3. Coaxial biopsy technique using quick core biopsy set. Top row shows 16-gauge guiding needle with stylet inside and thinner 18-gauge biopsy needle in "ready" position. On bottom row, biopsy needle is placed inside guiding needle after removing stylet and specimen notch is advanced (inset specimen notch). By fully pressing plunger, cutting cannula is fired and tissue sample is captured within specimen notch. 1 , guiding needle; 2 , plunger; 3 , specimen notch; 4 , cutting cannula; 5 , stylet of guiding needle.

selection in any given case depends on a number of factors, including the size and location of the target lesion, intervening structures in the planned biopsy path, status of the underlying lung, experience of the radiologist, and the estimated amount of tissue needed for diagnosis. Due to the unavailability of an onsite cytopathologist, we prefer to use coaxial core biopsy needles and routinely obtain $2-3$ tissue cores.

\section{Patient Positioning}

During a lung biopsy, the prone position is preferred as it allows the least chest wall motion with an added advantage of a comfortable "biopsy side down" post biopsy positioning of the patient (4). The supine position is associated with moderate chest wall motion, while the lateral decubitus position is associated with the maximum chest wall motion. However, patient positioning should be based mainly on lesion accessibility and the safest path to the lesion.

\section{Breathing Instruction}

Most upper lobe lung lesions can be targeted during gentle breathing and no special breathing instructions are required $(3,4)$. However, breath holding instructions 
are important during biopsy of lung lesions closer to diaphragm due to respiratory motion (Fig. 4) (3). Patients are explained to take small inspirations so that there may be minimal motion once a needle has passed through the pleura, as deeper inspiration will cause significant needle movement with greater chances of tearing the pleural surface $(3,6)$. Patients are instructed to hold their breath consistently (same degree of inspiration) each time during scanning or needle manipulation, so that the target lesion maintains a predictable position throughout the biopsy procedure (3). Sometimes, a patient may be asked to hold their breath differently to align the target lesion with the needle path. For example, during biopsy, the lesion has moved caudal to the needle path, and breath holding in expiration may help and vice versa (Fig. 5) (6). Although no definite breathing instructions have been described for the biopsy of mediastinal lesions, we have asked the patients for breath holding if we felt that with respiration the lesion could move or the lung may come in the path of the needle; otherwise, a biopsy was obtained in gentle breathing.

\section{Use of Intravenous Contrast}

For lesion localization, a non-enhanced CT alone is usually sufficient in most patients who have a recent diagnostic contrast-enhanced CT. However, occasionally, intravenous contrast agent administration may be required during the procedure to define the vascular structures in the anticipated needle path (Fig. 6).

\section{Locating the Needle Entry Site}

Table position of the most suitable axial image is noted for marking the biopsy plane. The patient is moved in the CT gantry at the same table position and this level is marked over the patient's skin with the help of a laser light emitted from the scanner (Fig. 7). To calculate the distance from the midline of the $\mathrm{CT}$ gantry to the anticipated skin

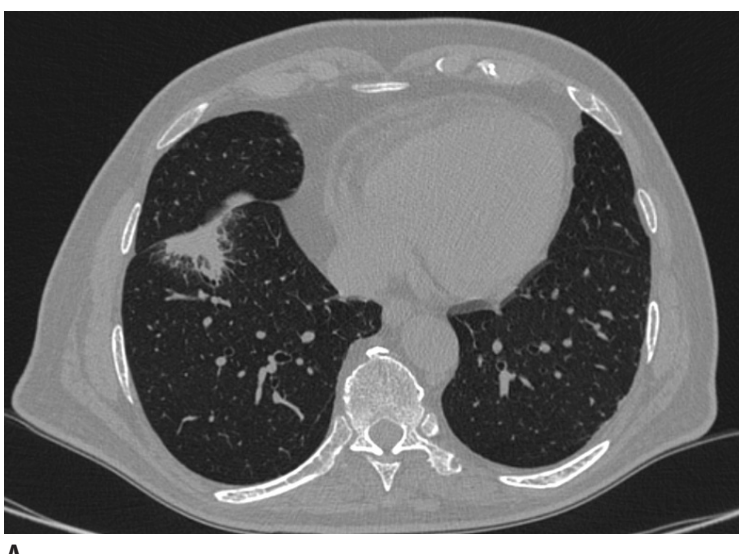

A

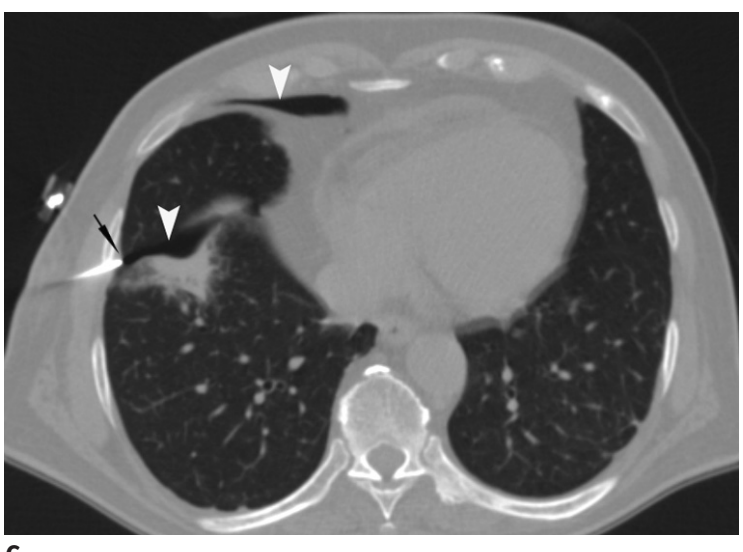

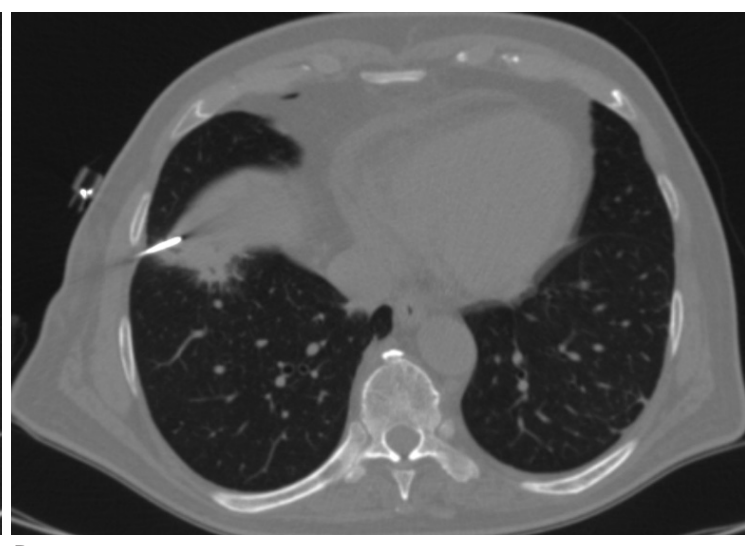

B

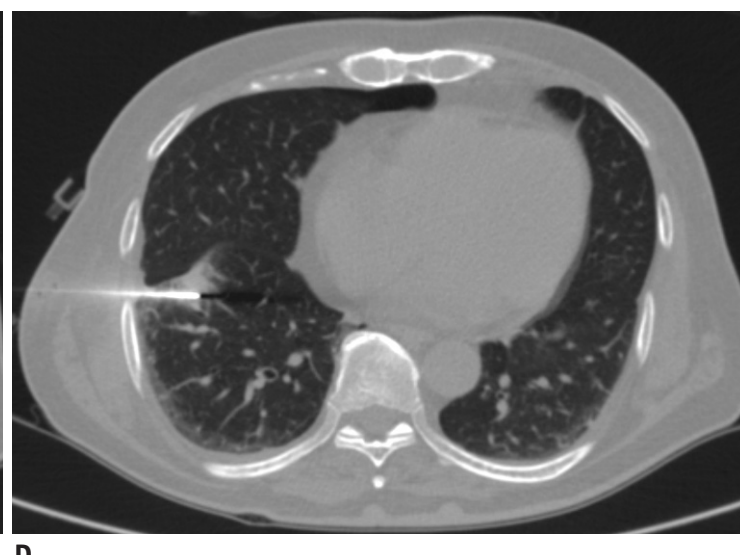

D

Fig. 4. Displacement of needle tip due to respiratory movement in lower lobe lesion.

A. Axial high resolution CT image of 72-year-old man shows irregular fissure based lesion in right lower lobe. B. Guiding needle was seen placed inside lesion. C. Subsequent scan showed that needle tip has slipped out of lesion due to excessive respiratory movement and lying in pleural space (black arrow). Presence of pneumothorax was also noted (arrowheads). D. Biopsy was completed after observing stable nature of pneumothorax. 


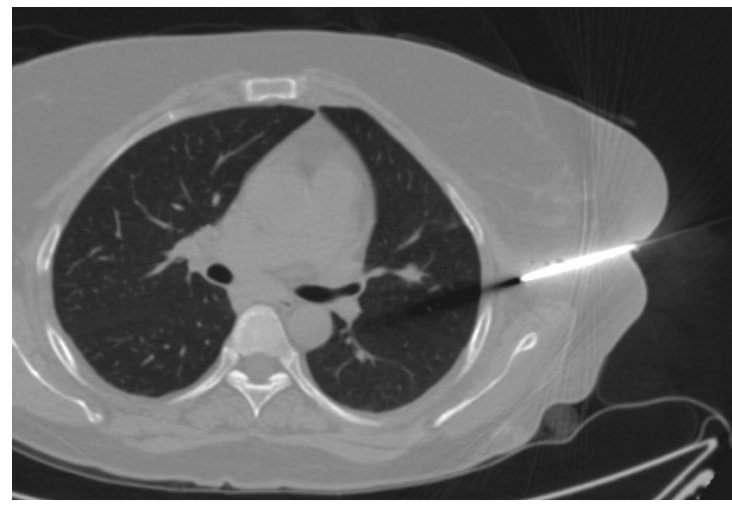

A

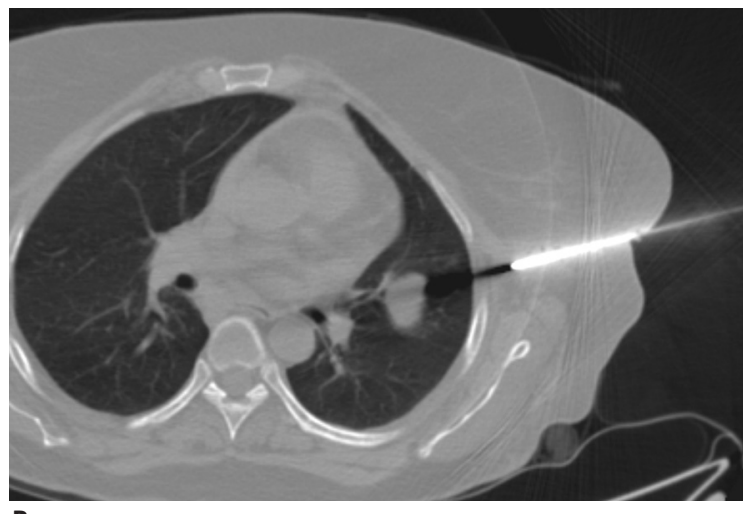

B

Fig. 5. CT images of 41-year-old woman with nodular lesion in her left lung.

A. Axial CT image taken during biopsy procedure shows that lesion has moved caudal to needle trajectory. B. Subsequently, patient was instructed to hold their breath in expiration and this maneuver again brought lesion within biopsy path.

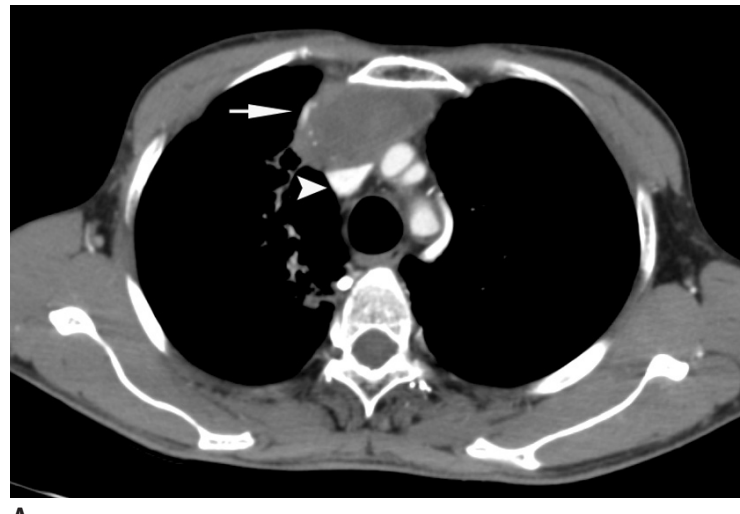

A

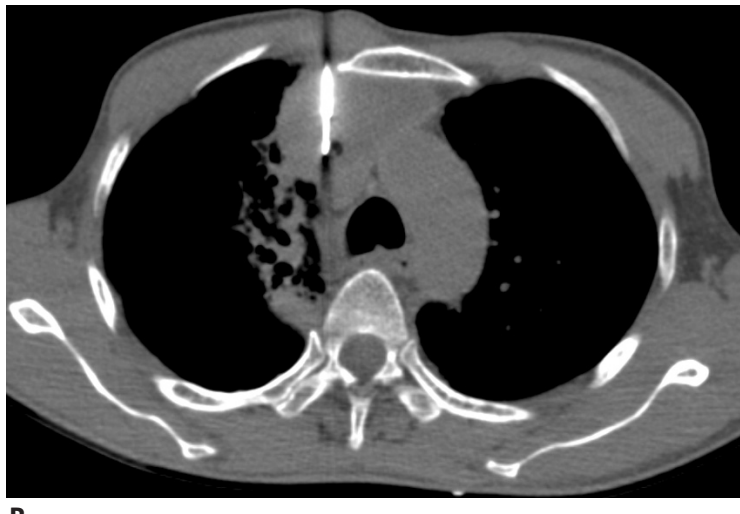

B

Fig. 6. Utility of intravenous contrast in delineation of vascular structures.

A. Contrast-enhanced CT of 50-year-old man shows anterior mediastinal mass and surrounding vascular structure including superior vena cava (arrowhead) and internal thoracic artery (arrow). B. Biopsy was obtained from right parasternal approach avoiding these vascular structures.

entry site and the lesion depth, the grid superimposition technique is used (1). The skin entry site is marked using a measuring scale and the laser light, in correspondence to midline (Fig. 7). The skin entry site may be marked with an inedible marker or, if inedible marker is not available, an impression may be created over skin with the back of the needle hub (Fig. 7). After cleaning the area, a small plastic marker or hypodermic needle is placed on the skin mark and a scan is obtained at that level for confirmation (Fig. 8) (7). Next, a local anesthetic is injected and if required, a small skin incision is made with a no. 11 scalpel.

\section{Sterile Drape as Needle Holder}

A sterile drape can be fashioned to hold the needle and to maintain the desired needle angulation. This technique is especially useful when skin to pleura depth is short, resulting in poor fixing force (Fig. 9) (7). A sterile drape may also be used to support the needle when needle entry ranges from the lateral aspect of the chest wall in the supine or prone position (Fig. 10).

\section{Needle Manipulation}

During lung biopsies, the guiding needle is placed in the soft tissue of the chest wall and all necessary adjustments should done to align the needle perfectly with the target lesion prior to piercing the pleura (Fig. 5) (6). All the needle manipulations, even those in the chest wall, should be performed with the patient in the designated breath hold. The anticipated path of the needle is traced on the CT console by extrapolating the needle towards the lesion or it may be depicted by the beam hardening artifact produced by the needle tip. Thereafter, the needle is advanced in one stroke into the lung till the lesion margin in peripherally situated lesions, or at least $1-2 \mathrm{~cm}$ inside the lung. Leaving at least $1-2 \mathrm{~cm}$ needle inside the lung parenchyma avoids slipping of the needle into the pleural space during respiratory motion and prevents needle tip laceration of pleura. This is especially important in cases of lower lung 


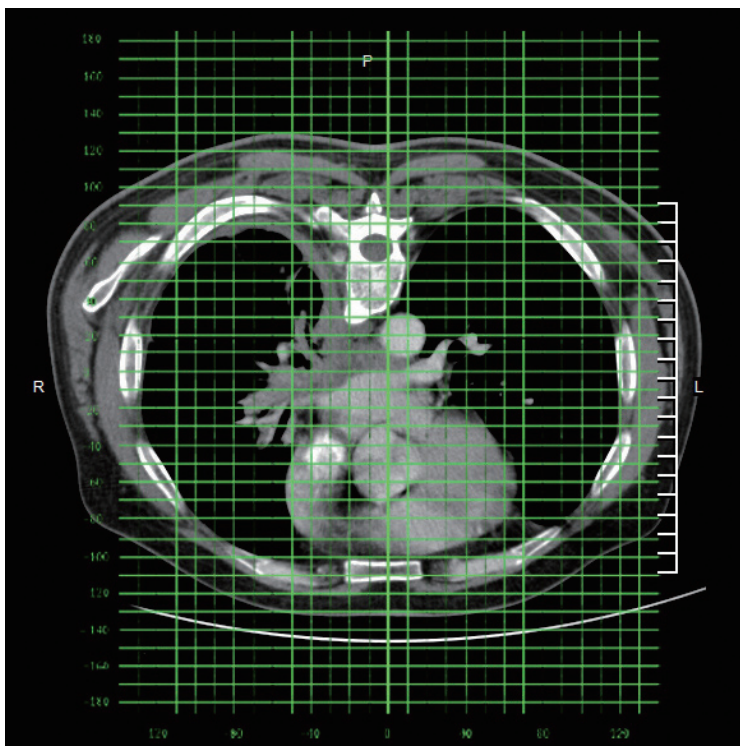

A

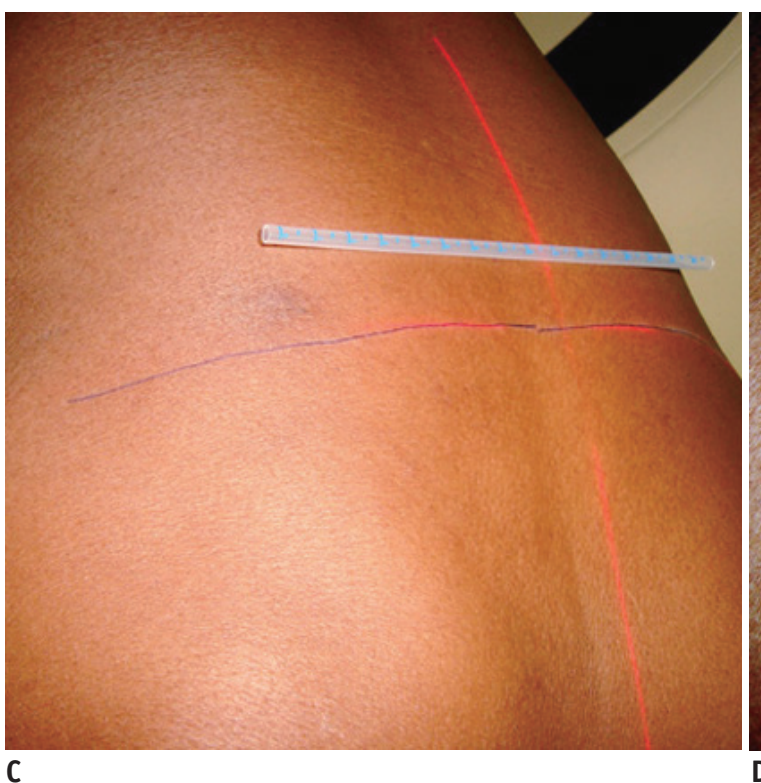

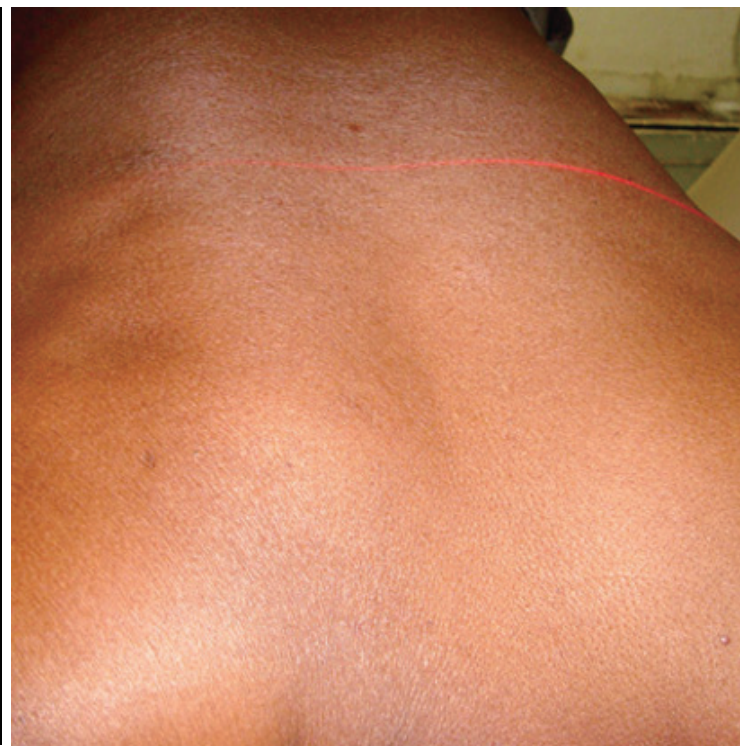

B

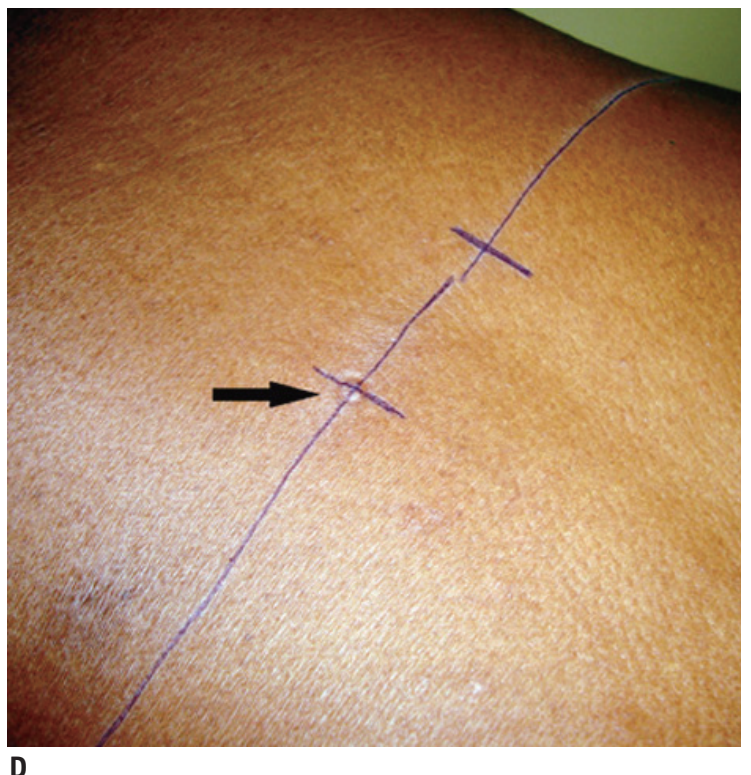

Fig. 7. Technique of marking skin entry using grid method.

A. Axial CT image of 60-year-old man showing infiltrative mass. Skin entry site selected on CT console and its location was noted in relation to superimposed grid lines. B. Firstly, axial level was marked by moving patient in CT gantry at same table position. C. Lateral distance is measured from midline laser light in alignment with central grid line. D. After marking skin entry site, impression was made over skin by pressing back of needle hub (arrow), since it remains visible even if ink is erased during cleaning process.

areas where the target moves significantly and the minimum needle indwelling depth has been suggested to be $1.5 \mathrm{~cm}$ for the lower lobes and $1.0 \mathrm{~cm}$ for the upper lobes (6). After entry into lung, the needle should be left to rock freely with respiration and should be touched only during the designated breath hold.

While ultrasound guided lung biopsy may be more suitable for peripheral lung masses or some mediastinal lesion provided an adequate acoustic window is available, smaller subpleural lesions usually require CT guidance.
Small subpleural lesions are also difficult to biopsy because a short needle length inside the lung is unstable and can be easily dislodge during respiratory motion, resulting in tearing of the pleural surface (Fig. 11) (8). A tangential route has been preferred by some authors rather than a right angle path for sampling the subpleural lesions, as it offers greater needle stability and easier needle correction (Fig. 12) (9). Moore (6) has described a technique for sampling tiny subpleural nodules in which a perpendicular direct puncture is planned. He preferred a single needle 
technique and after the lesion is transfixed, the needle is passed through the whole diameter of the nodule and the patient is allowed to breathe. Then, in a single breath hold, the needle tip is pulled back to the center of the nodule, and the deep half of the nodule is repeatedly aspirated by using a pass length equal to the radius of the lesion. By following this method a longer length of needle will stay inside the lung parenchyma during breathing, which will provide a better anchoring and prevent needle dislodgment. To employ this technique with a coaxial system, first the guiding needle is advanced through the nodule and the patient is allowed to breathe. While sampling, the patient is asked to hold their breath and the guiding needle is withdrawn into the lesion. Sampling is then performed with the biopsy needle. And again, the guiding needle is readvanced through the nodule and the patient is allowed to breathe quietly.

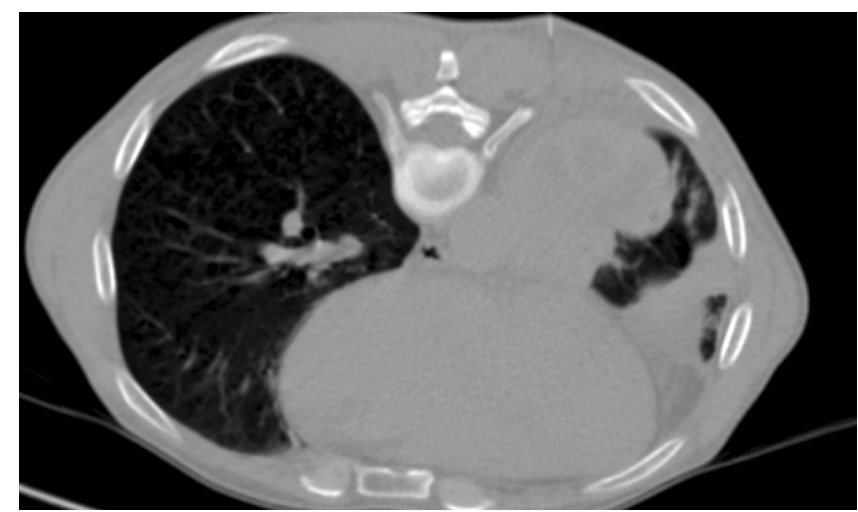

Fig. 8. Axial CT image of 58-year-old man with left lower lobe mass. Hypodermic needle is placed to check accuracy of skin entry site before insertion of guiding needle.
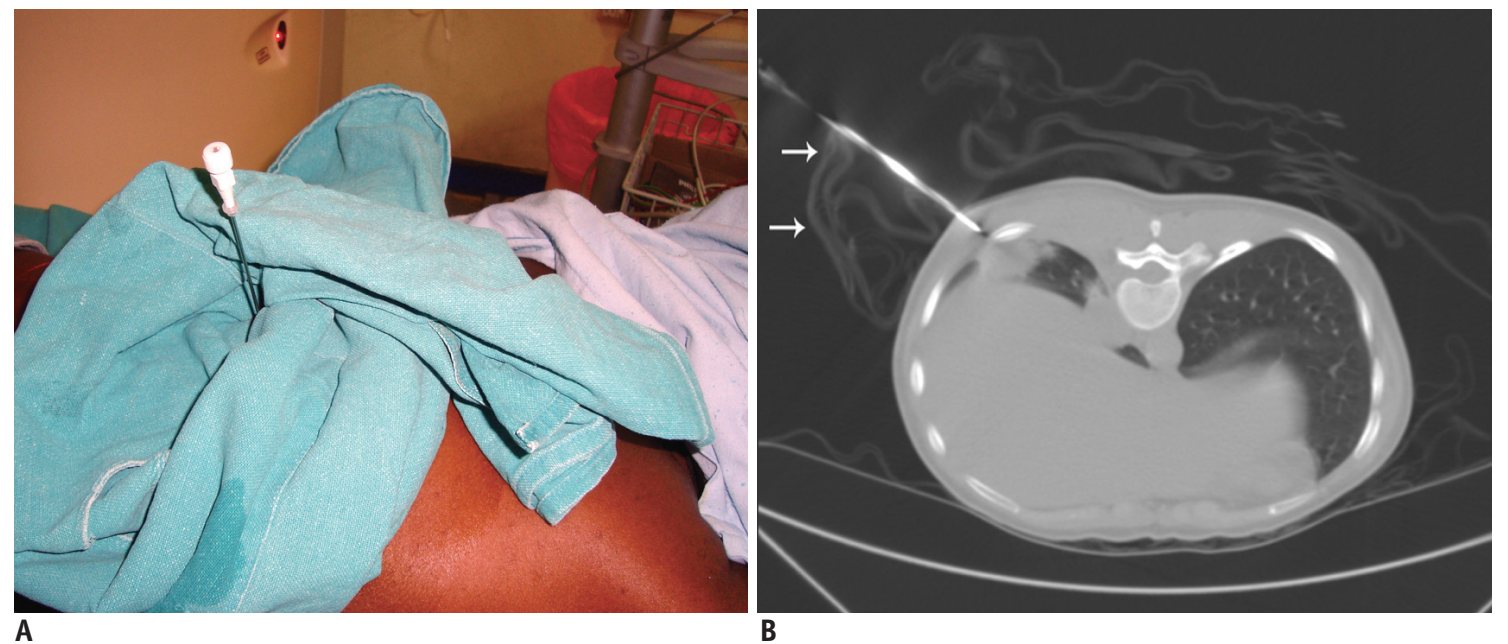

Fig. 9. Use of sterile drape as needle holder.

A. Photograph of 26-year-old woman who presented with right lower lobe lung mass. Sterile drape was fashioned to support needle in desired angulation. B. Axial CT image shows sterile drape (arrows) holding needle in alignment with mass. 


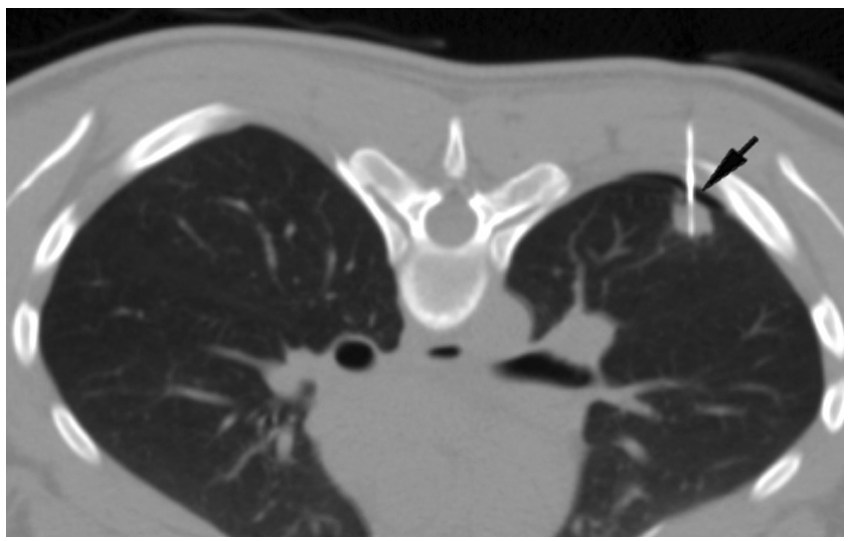

Fig. 11. Axial CT image of 23-year-old woman with left lower lobe subpleural nodule. After guiding needle was inserted into lesion, pneumothorax (black arrow) developed by time biopsy needle was exchanged with stylet. Although tip of guiding needle was advanced inside lesion margin, small needle length inside lung resulted in slipping of needle tip in and out of lesion during respiration motion, resulting in pneumothorax. However, biopsy was obtained successfully under stable pneumothorax.

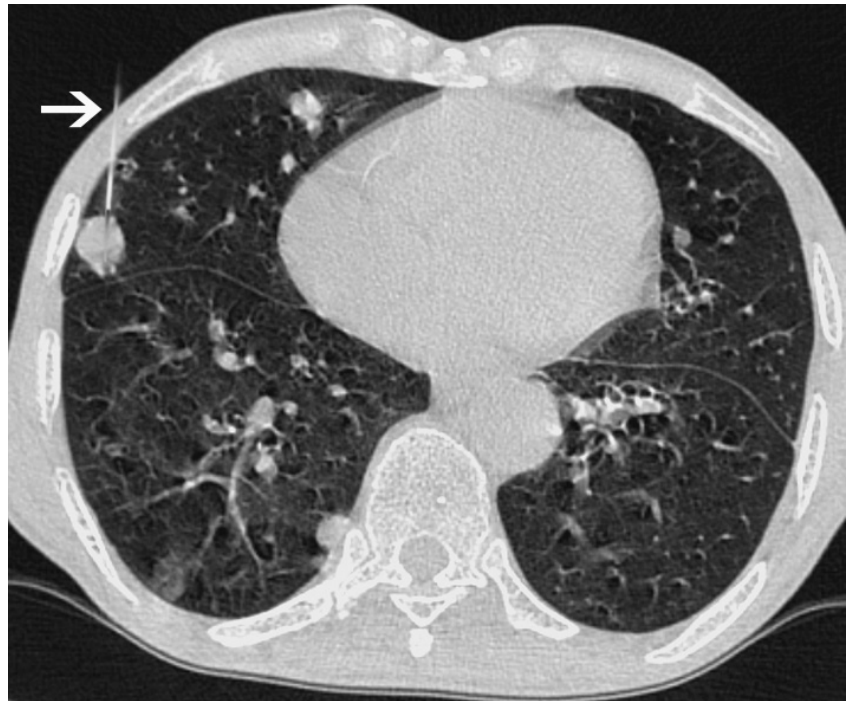

Fig. 12. Axial CT image of 74-year-old male showed multiple nodular lesions in both lungs. Tangential approach was used to sample subpleural nodule in right middle lobe (arrow). Biopsy results suggested evidence of squamous cell carcinoma.

from the skin to the lesion and the latest acquired scan should always be monitored for further planning as there are various types of movements which can change lesion location during the procedure (patient movement, cardiac and respiratory motion, development of pneumothorax). Hence, in many cases, a corrective manipulation of the needle is required either due to the change in the lesion location or if the initial puncture is imperfect. The "bevel steering" technique has been used with thinner gauge flexible needles having a single bevel tip $(4,6)$. This technique is based on the principle that a beveled needle has the tendency to drift in the direction away from the beveled side because the bevel side faces more tissue resistance (4). So to direct a beveled needle towards the lesion, it should be partially withdrawn and readvanced after rotating the bevel side away from the lesion. The skin and soft tissue adjacent to the puncture site can also be dragged away from the lesion to torque the needle further, hence increasing the amount of steering.

To correct the course of relatively stiff needles like larger gauge needles or a guiding needle of the coaxial systems, the needle is partially withdrawn and the needle tip is redirected by pushing the hub in the desired direction while applying fulcrum-like pressure at the skin surface (6). Then, the needle is re-advanced towards the lesion while maintaining it in the desired angulation. While manipulating the needle in the lung, withdrawal and readvancement of the needle tip through the pleura should be avoided (6). To correct cranio-caudal misalignment of the needle tip in the lung, varying the designated breath hold may bring the lesion in the needle path. For example, if the lesion is cranial to the needle tip, the partial withdrawal of the needle in the periphery of the lung followed by a larger inspiration and re-advancement may correct the position (6).

Sometimes, despite careful needle insertion, the guiding needle of a coaxial system reaches slightly outside the margin of the lesion instead of hitting it. In such cases, a small amount of course correction in cranio-caudal or medial-lateral direction can be achieved at the time of sampling (6). The hub of the guiding needle pushed in the desired direction, followed by sampling with the inner biopsy needle is performed while maintain the guiding needle in the desired angulation (Fig. 13). This maneuver is helpful for bringing smaller lesions in the biopsy path and sometimes for avoiding vascular structures (7). Similarly, small tilting movements may be done while cutting multiple tissue cores from a lesion, and specimens is obtained from different areas of the same lesion.

\section{Oblique Approach}

If a bony structure is in the way of a planned trajectory, various maneuvers can be helpful such as changing the position of the patient's arm, taking a more medial or lateral path, rotating the patient, puncturing the patient from the opposite side if feasible, and varying the designated respiration (6). Lesions which cannot be targeted due to an intervening bone or vascular structure can also be 


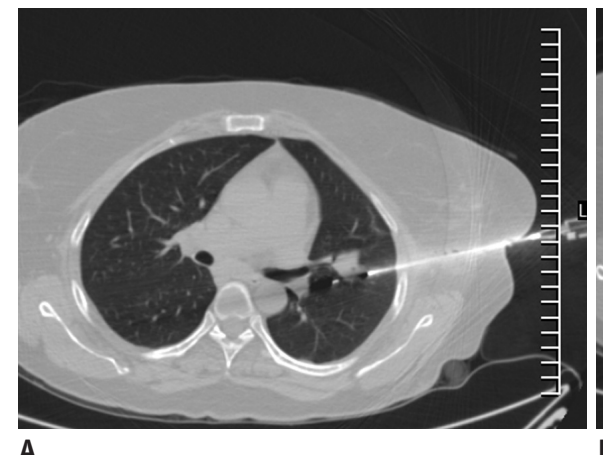

A
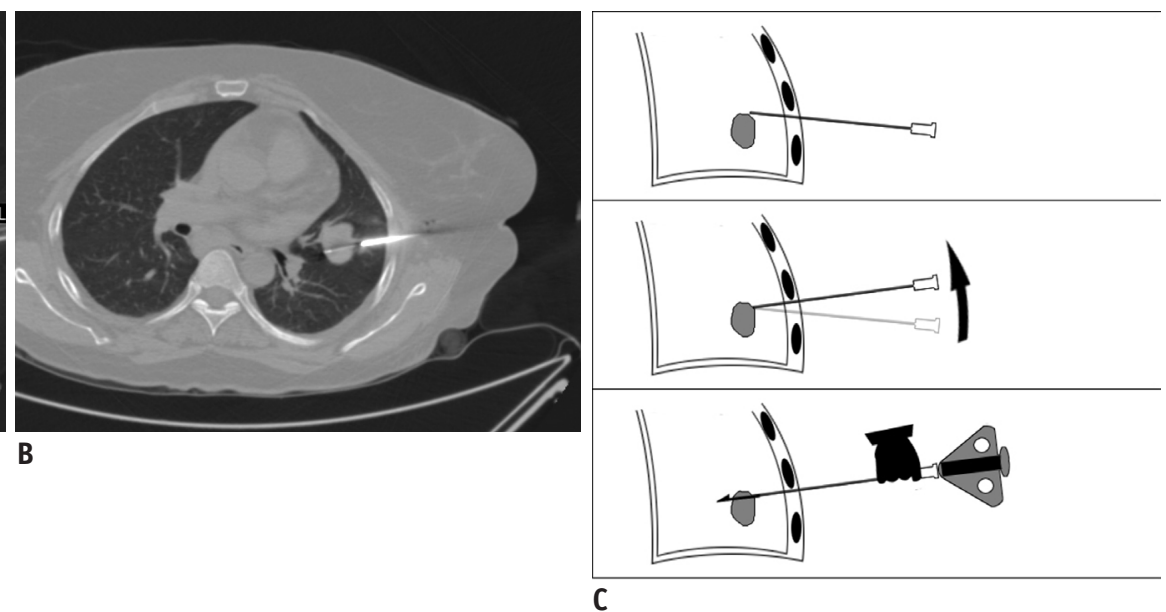

Fig. 13. Needle manipulation at time of sampling.

A. Axial CT image of 41-year-old woman showed nodular lesion in left lower lobe. After advancing biopsy needle, specimen notch just missed lesion. B. Specimen notch was withdrawn and whole coaxial assembly was angulated caudally. Moreover, while maintaining guiding needle in caudal angulation, specimen notch was again advanced. Now CT image showed specimen notch well inside lesion. C. Line diagram demonstrating manipulation technique.
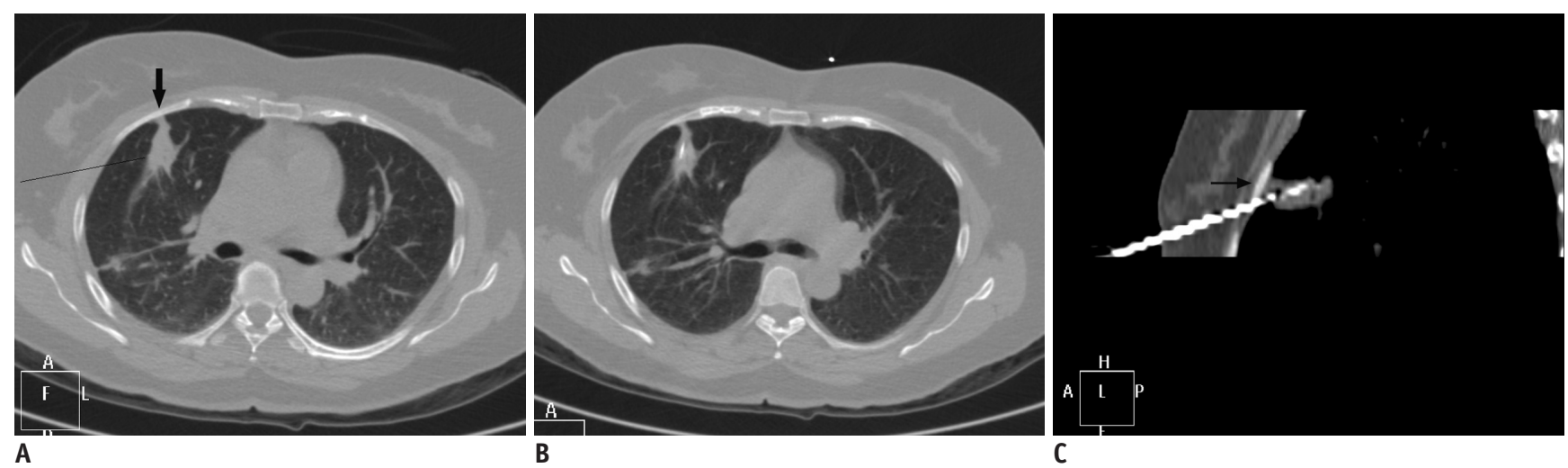

Fig. 14. Oblique approach.

A. Localization CT scan of 57-year-old woman with nodular lesion having irregular margins. Anterior end of rib (black arrow) was blocking anterior approach. Alternative approach (black line) is very long and through soft tissue of breast. B. Hence lesion was targeted using oblique approach and minimal lung parenchyma was traversed. Specimen notch is seen in lesion center. C. Sagittal reformatted image demonstrated oblique course of needle from inferior rib margin (arrow) into lesion. Biopsy was suggestive of chronic interstitial fibrosis.

approached using angled insertion of the biopsy needle in the z-axis (Fig. 14). Simultaneous angling of the CT gantry in the plane of the needle will help in the visualization of the entire needle and the target lesion in one CT section (Fig. 15) (11). Coronal and sagittal multiplanar reconstructions are very valuable especially while employing an oblique approach and obtaining help in deciding the required angulation and choosing the skin entry site.

\section{Scan Parameters}

During the procedure, low dose 2.5-5.0 mm axial scans are usually sufficient to monitor the advancement of the needle and for the detection of any complications. Decreasing the tube current to 30-50 mAs resulted in a marked reduction of the total radiation dose to the patient (7). During all check scans, at least one superior and one inferior CT section to the needle tip was required.

\section{CT Fluoroscopy}

CT fluoroscopy is a technical advancement which enables real-time visualization of a lesion during needle manipulation (Fig. 16) (12). This technique is especially useful for targeting small lung lesions, juxtraphrenic lesions, and patients with poor breath holding capacity. CT fluoroscopy is more accurate than conventional CT in diagnosing pulmonary lesions with a significant reduction in complication rates (12). Additional advantages are the simplification of the biopsy process and decreased procedure time. However, this technique is associated with a small radiation to the operator and the mean estimated 
effective doctor dose ranging from 0.025 to $0.054 \mathrm{mSv}$ per procedure $(12,13)$. Although CT fluoroscopy is available on most of the newer CT scanners, this facility is not available on many of the older CT scanners.

\section{Anatomical Considerations for Different Approaches}

Parasternal Approaches to the Mediastinal Lesions

The parasternal approach is used for the biopsy of anterior and middle mediastinal lesions when the lesion

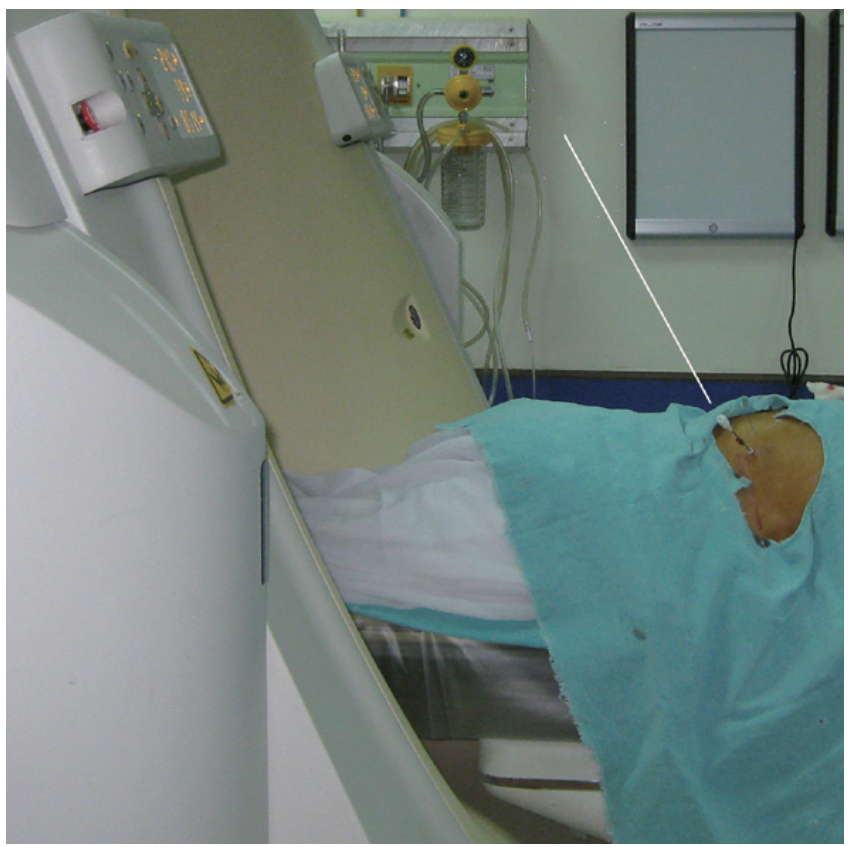

Fig. 15. Photograph taken during biopsy of 68-year-old woman with lower lobe lung mass. Oblique approach was used to avoid rib and $\mathrm{CT}$ gantry was also tilted (white line denotes plane of angulation). can be targeted from the lateral margin of the sternum (5). In this approach, the needle was inserted directly into the target lesion or through the intervening fat. The patient is usually placed in the supine position; however, sometimes the lateral decubitus position may be helpful for creating a safe window. The internal thoracic vessels should always be identified as they are located lateral to sternal margin and inadvertent injury may result in hematoma formation. In most cases, a needle is inserted close to the lateral margin of the sternum and medial to these vessels (Fig. 17). Sometimes, needle could be inserted lateral to the internal

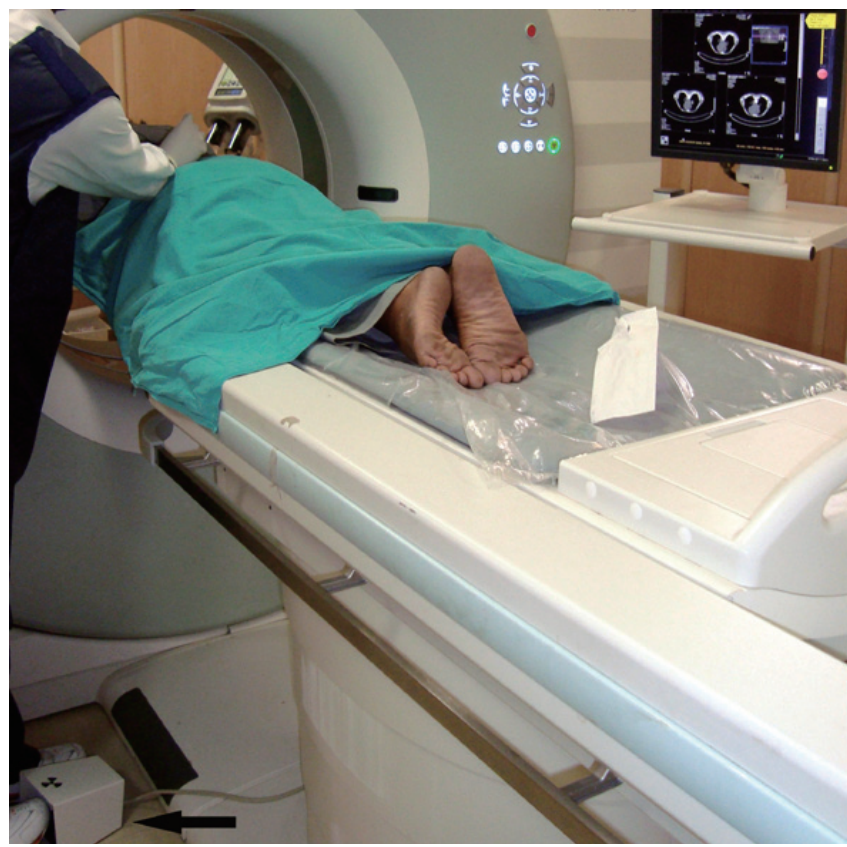

Fig. 16. Photograph of CT fluoroscopy unit taken during biopsy of lung nodule in 60-year-old man. Operator manipulated needle and visualized needle movement in real time on CT monitor. Foot switch (arrow) was pressed to obtain CT sections.
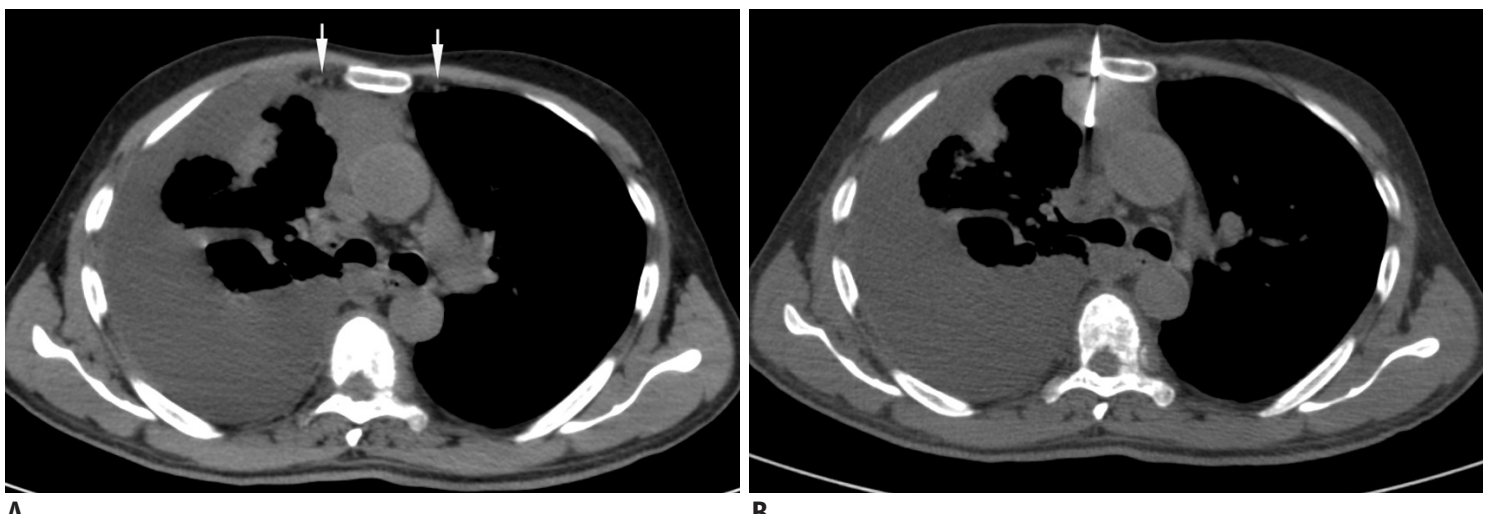

A

B

Fig. 17. Anterior parasternal approach.

A. Localization non-enhanced CT image of 59-year-old man with anterior mediastinal mass and right pleural effusion. Internal thoracic vessels were seen even without contrast enhancement (arrows). B. Biopsy was performed from right parasternal approach by placing needle just lateral to sternal margin. Biopsy was suggestive of colloid carcinoma. 
thoracic vessels if the lesion or the mediastinal fat was touching the anterior chest wall sufficiently lateral to the vessels (Fig. 18). Moreover, the degree of contact between the mediastinum and the parasternal chest wall may vary with breathing during the biopsy procedure, resulting in the inadvertent transgression of the pleura or lung (Fig. 19).

\section{Paravertebral Approach to the Mediastinal Lesions}

The paravertebral approach is usually used for obtaining a biopsy from the middle, posterior mediastinal and subcarinal lesions (5). The needle is advanced through the space between the endothoracic fascia and parietal pleura, usually from the right side. The presence of the

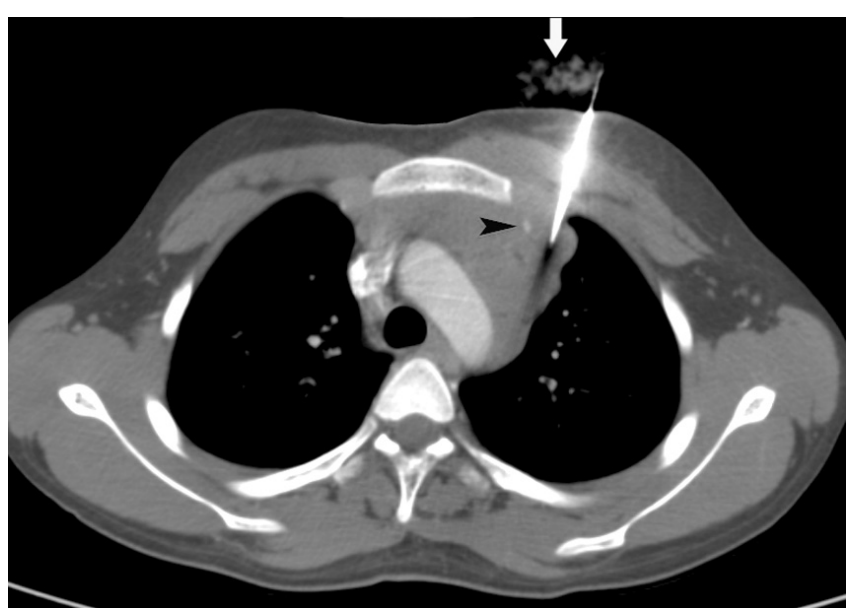

Fig. 18. Axial CT image of 18-year-old male that showed multiple mediastinal nodes. Biopsy was obtained from left parasternal approach lateral to internal thoracic artery (arrowhead) as lymph nodal mass was touching chest wall sufficiently to allow safe access lateral to internal thoracic vessels. To maintain needle in proper alignment wet gauze piece was placed (arrow). Biopsy results suggested Hodgkin lymphoma. aorta makes this approach difficult from the left side. The patient may be positioned in the prone, prone oblique, or lateral decubitus position. In some patients, the space between the endothoracic fascia and parietal pleura is wide enough for the direct advancement of the biopsy needle (Fig. 20). However, in others, this extrapleural space needs to be sufficiently widened by injection of saline solution to create a window for needle entry (Fig. 21). Advancing the needle from the paravertebral approach without displacing the parietal pleura off the spine, may lead to inadvertent transgression of pleura and lung. In addition, the paravertebral approach has the potential risk of injury to the esophagus, azygos vein, paravertebral vessels, intercostals vessels and nerves, as well as the spinal and vagus nerves. Other approaches described for mediastinal lesions are transsternal, suprasternal and the subxiphod approach; however, we rarely employed them at our institution.

\section{Transpulmonary Approach to the Mediastinal Lesions}

A transpulmonary path is employed when a mediastinal lesion could not be approached by the extrapleural route (Figs. 22, 23) (5). Patient positioning is achieved based on lesion accessibility and other precautions are same as a lung biopsy. The needle passes through the lung parenchyma and two layers of visceral pleura.

\section{Approach to Lung Lesion}

Generally the shortest intercostal route is taken during lung biopsies, avoiding fissures, bullous lesions and emphysematous areas (9). Sometimes, lung masses have associated collapsed or consolidated lung. Use of
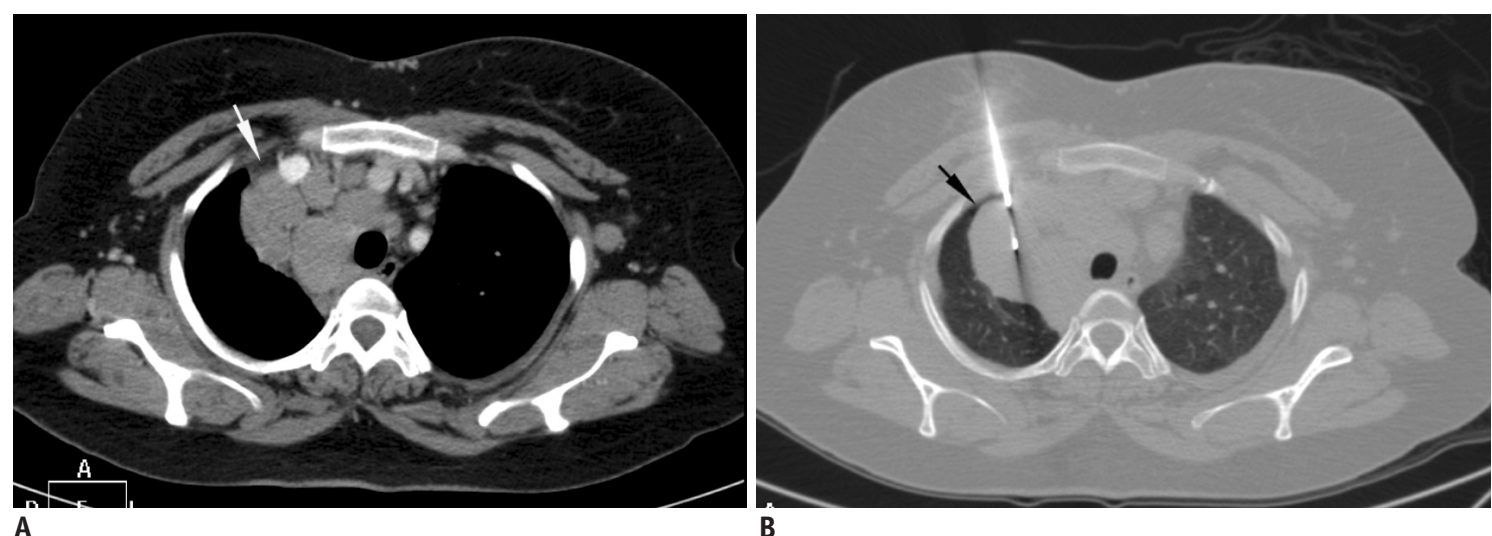

Fig. 19. Inadvertent pneumothorax during mediastinal biopsy.

A. Localization contrast-enhanced CT scan of 40-year-old woman with multiple mediastinal nodes. Safe window for biopsy was present lateral to right brachiocephalic vein through mediastinal fat (white arrow). B. CT image after advancing needle showed air (black arrow) in path of needle with separation of mediastinal fat from anterior chest wall. Pneumothorax had occurred, either due to inadvertent transgression of interposed parietal pleura or variable respiration bringing aerated lung in path of needle. 

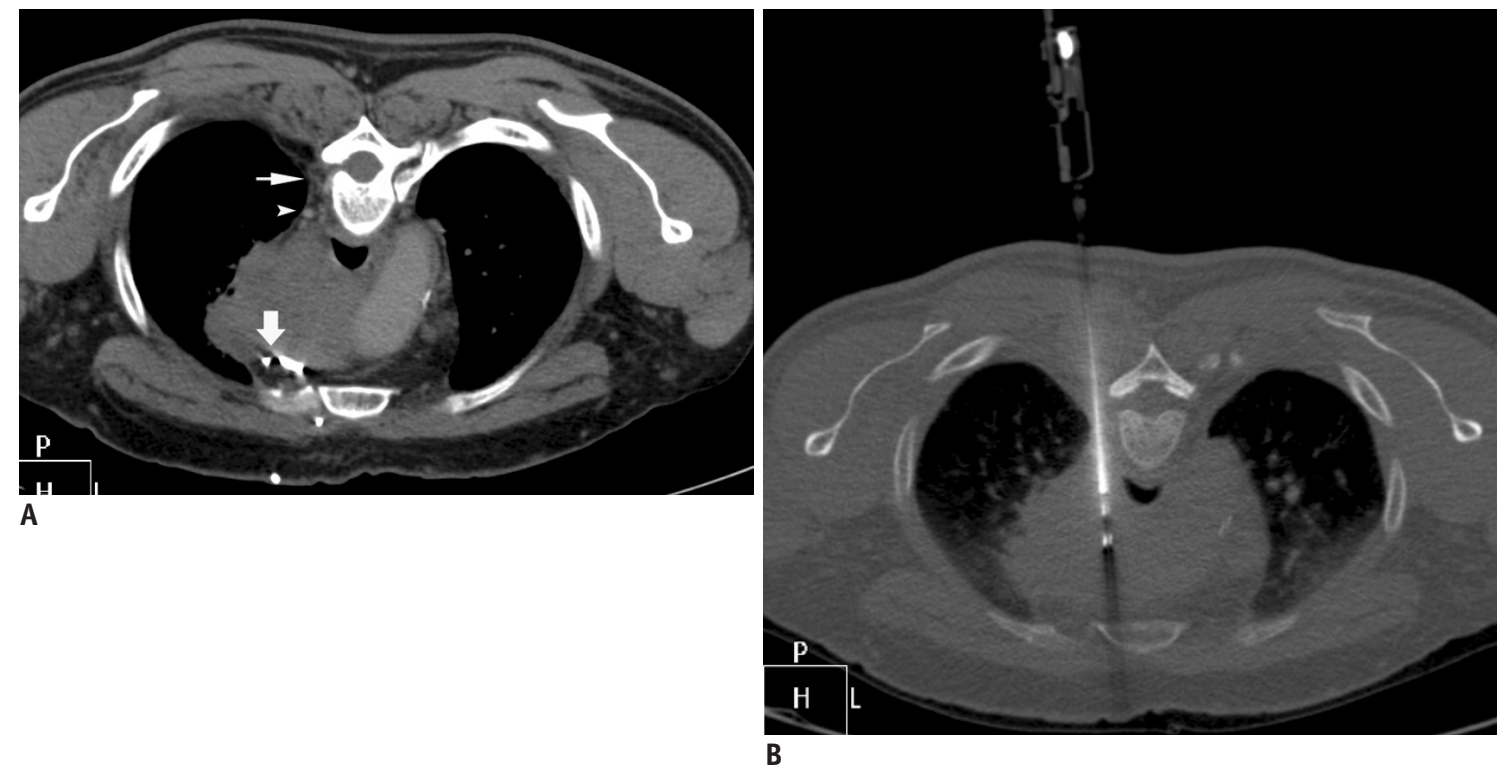

Fig. 20. Posterior paravertebral approach.

A. Prone CT scan of 57-year-old man with large mediastinal mass. Window for biopsy through right paravertebral extrapleural approach was available due to presence of paravertebral fat (thin arrow). Small venous channel (arrowhead) was noted close to needle path. Anterior approach was not possible due to multiple vascular channels (thick arrow). B. Needle was safely advanced from side of vertebra into mass without injuring small vein.

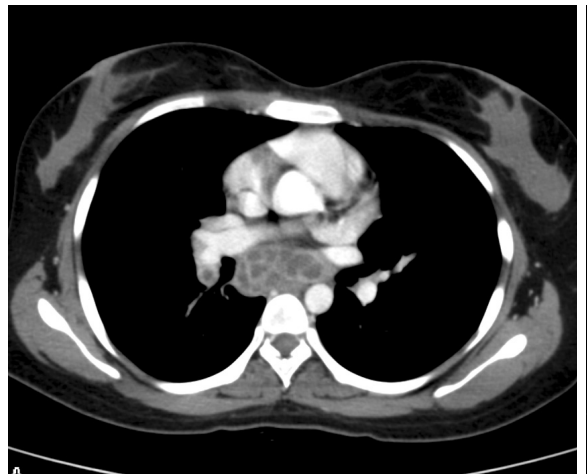

A

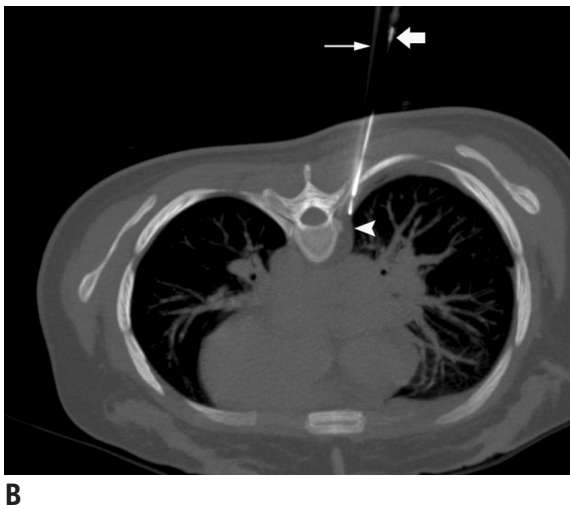

B

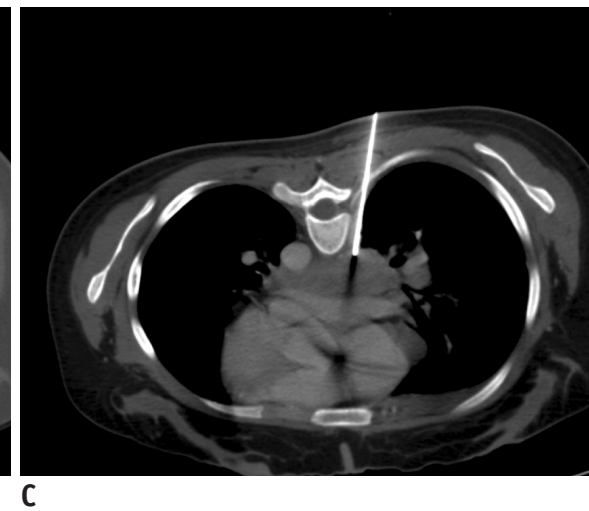

Fig. 21. Posterior paravertebral approach with saline instillation technique.

A. Contrast-enhanced CT scan of 21-year-old woman with multiple necrotic subcarinal nodes showed no extrapleural paravertebral window to lesion. B. 22-gauge chiba needle (thin arrow) was advanced into paravertebral extrapleural space and saline solution was injected. After widening extrapleural space, safe window (arrowhead) was created, and guiding needle (thick arrow) was advanced through this space. C. Final positioning of guiding needle was made to edge of nodal mass. Biopsy and culture reports were suggestive of granulomatous lesion due to mycobacterium tuberculosis.

intravenous contrast is helpful in this setting as a mass usually enhances less than the collapsed lung tissue, and vascular markings of the lung parenchyma are not seen coursing through the mass (Fig. 24). If a non-aerated route to a lung lesion is possible, it is preferable as it reduces the chances of pneumothorax (6). It may be through an area of contact of mass with the pleura or mediastinal fat (Figs. $25,26)$. From a cavitary lesion, biopsy is obtained from the lesion wall; however, in the presence of internal content like suspected fungal ball, biopsy can be obtained from the cavity wall as well as from the ball-like lesion (Fig. 27) (14).

\section{Complications}

Despite observing all precaution complications may occur, hence early detection of complications and timely management are important. The patient's vitals and the latest acquired scans are carefully monitored. Pneumothorax, pulmonary hemorrhage, hemothorax and chest wall hematoma are the most commonly encountered complications $(2-4,15,16)$. Other infrequently reported complications are hemomediastinum, cardiac tamponade, air embolism, vasovagal reaction, surgical emphysema, and 
tumor seeding $(3,16)$.

The reported incidence of post-biopsy pneumothorax ranged from $27 \%$ to $54 \%$ in most of the large series (Figs. 28, 29) $(1,15)$. Most of these cases were managed

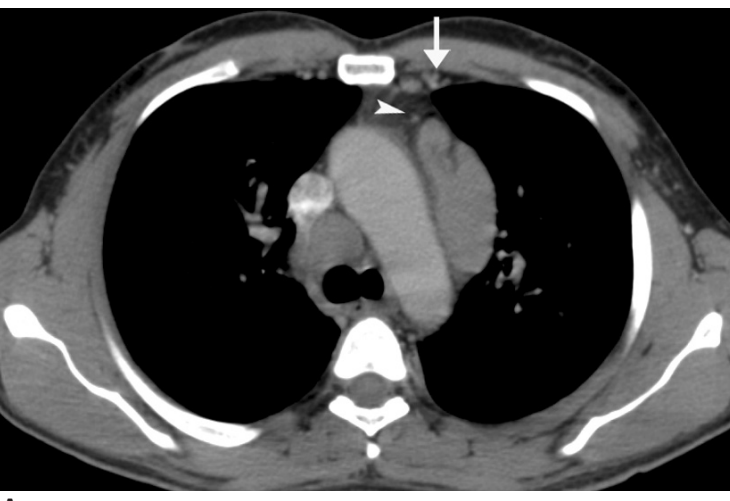

A conservatively; however, about $3-15 \%$ of patients required a 6 to 10.3-F chest tube placement if pneumothorax became symptomatic or continued to increase $(1,15$, 16). In our experience, we also found that the majority of

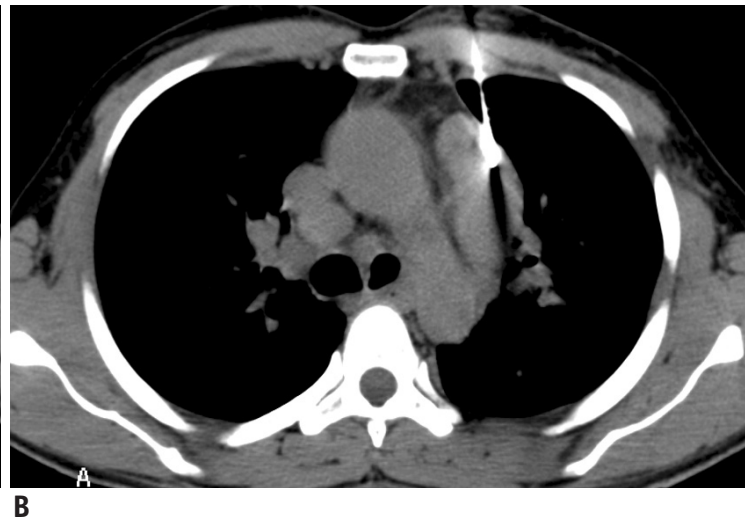

Fig. 22. Anterior transpulmonary approach to mediastinal lesions.

A. Localization CT scan of 38-year-old man with multiple mediastinal nodes. Left internal thoracic vessels (arrow) and tiny vessel (arrowhead) just anterior to preaortic nodal mass were precluding extrapleural approach through mediastinal fat. B. Guiding needle was advanced through lung into lesion. Biopsy results were suggestive of sarcoidosis.
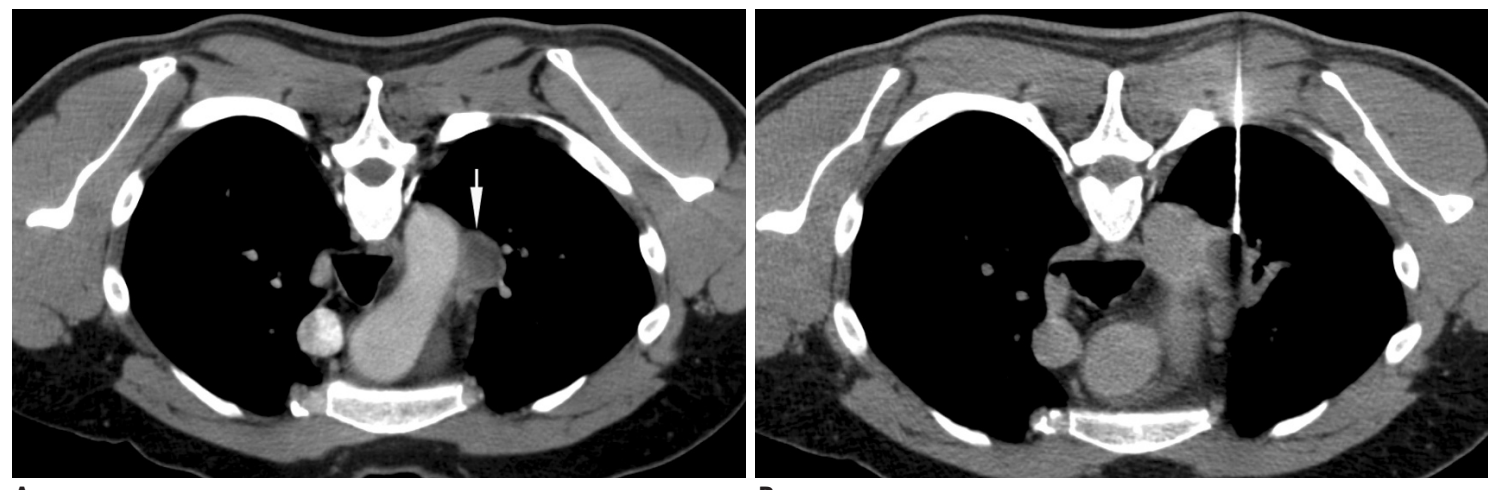

A

B

Fig. 23. Posterior transpulmonary approach to mediastinal lesions.

A. Prone contrast-enhanced CT scan of 42-year-old man showed necrotic mediastinal node (arrow) just lateral to aortic arch and no extrapleural approach was possible. B. Guiding needle was advanced to lesion margin through lung parenchyma.

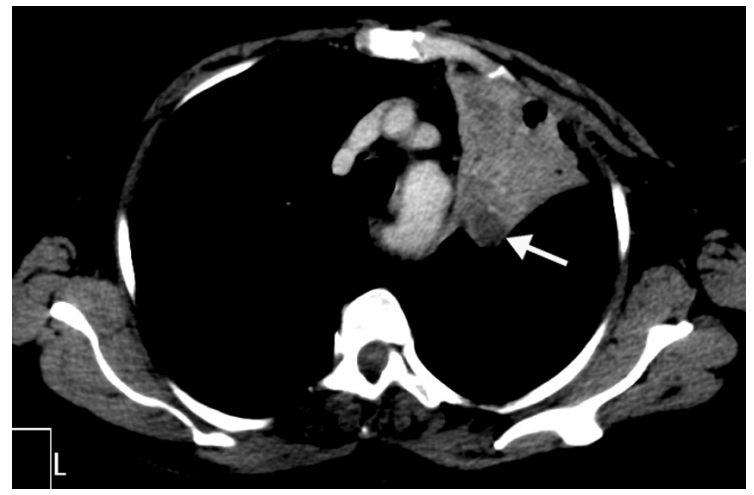

A

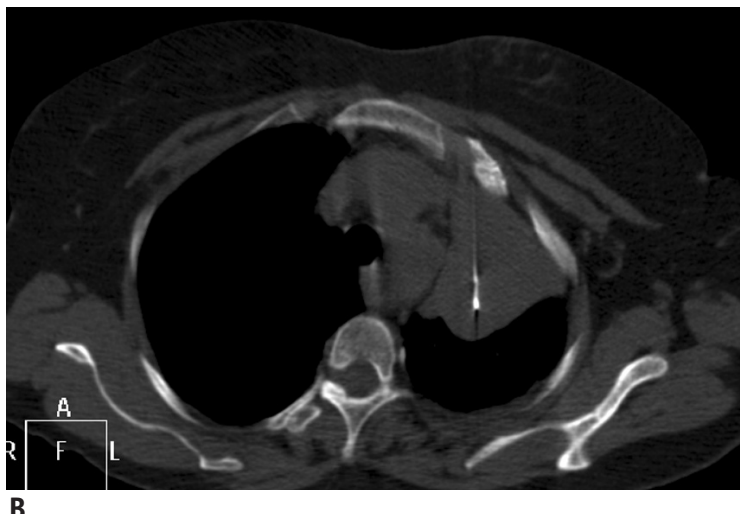

B

Fig. 24. Use of intravenous contrast to differentiate mass lesion from collapsed lung.

A. Contrast-enhanced CT scan of 71-year-old woman showed collapsed left upper lobe and less enhancing lesion (arrow) in posterior part of collapsed lung. B. Needle was advanced through collapsed lung into lesion. Without contrast, it was difficult to differentiate mass from adjacent collapsed lung. 

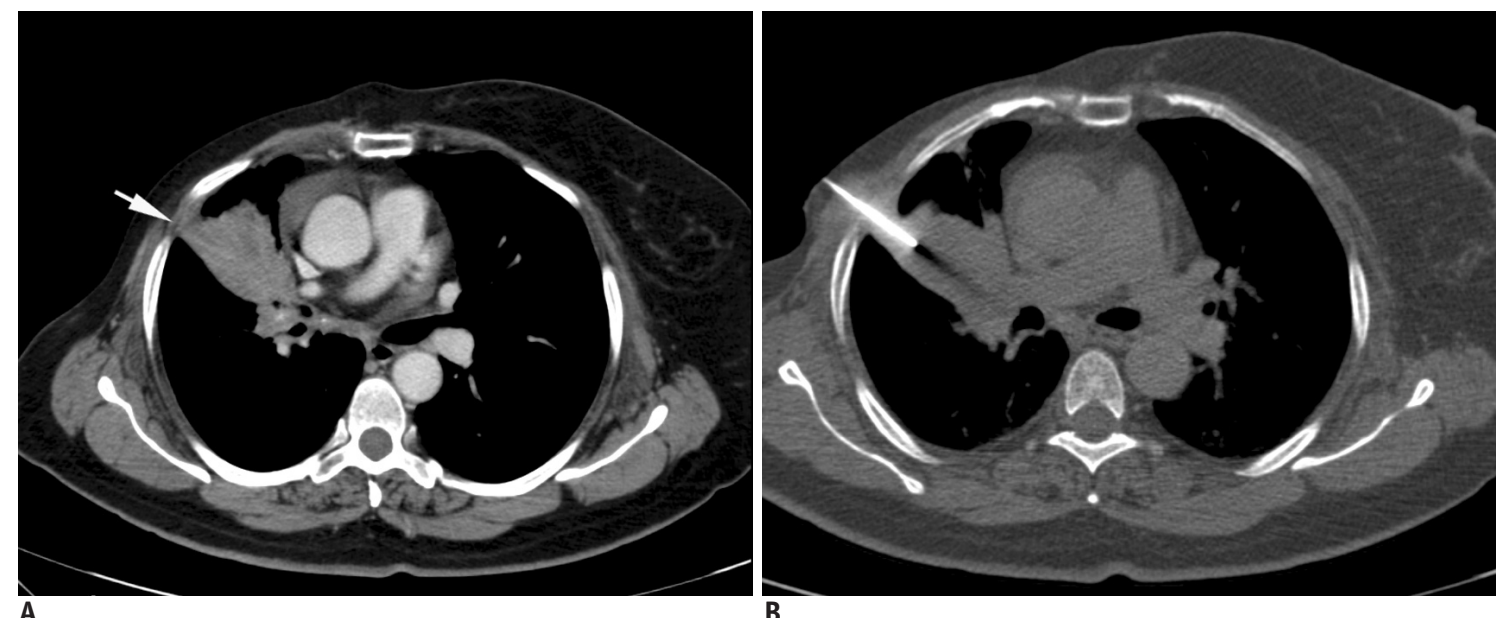

Fig. 25. Lung biopsy through non-aerated route from area of pleural contact.

A. Contrast-enhanced CT scan of 61-year-old woman who was operated on to remove carcinoma of right breast and received radiotherapy. Mass lesion was seen in right upper lobe with small contact between mass and lateral pleura (arrow). B. Guiding needle was advanced through this area of contact without transgressing aerated lung. Biopsy results were suggestive of metastatic disease.

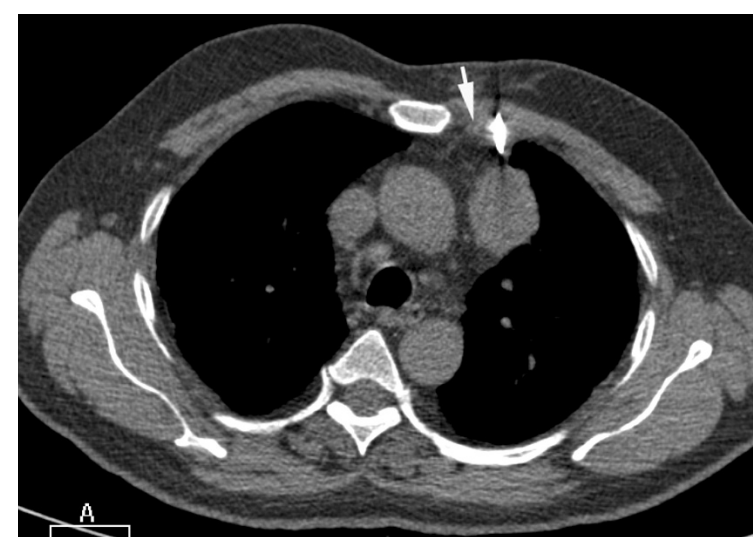

A

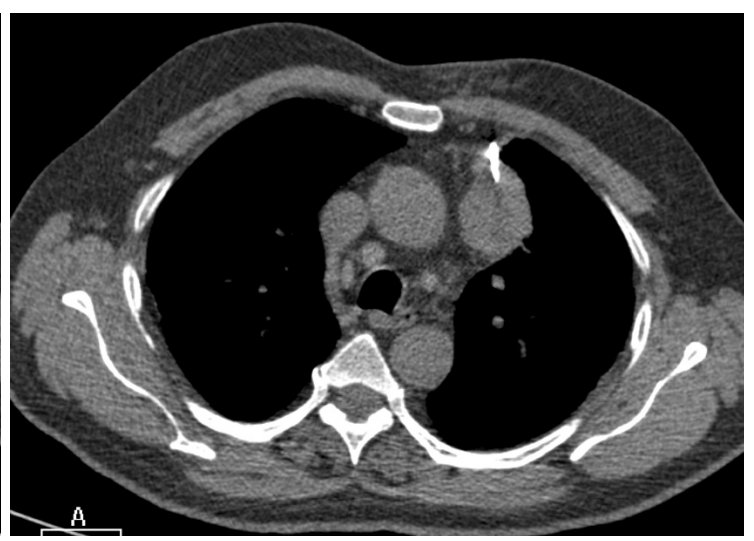

B

Fig. 26. Lung biopsy through non-aerated route using mediastinal fat as window.

A. Axial CT image of 68-year-old man shows left upper lobe mass which was abutting mediastinal fat. Biopsy needle was advanced from left parasternal approach lateral to internal thoracic vessels (arrow) using mediastinal fat as window. B. CT image at caudal level showed needle entering into mass without transgression of aerated lung. Biopsy results were suggestive of adenocarcinoma.

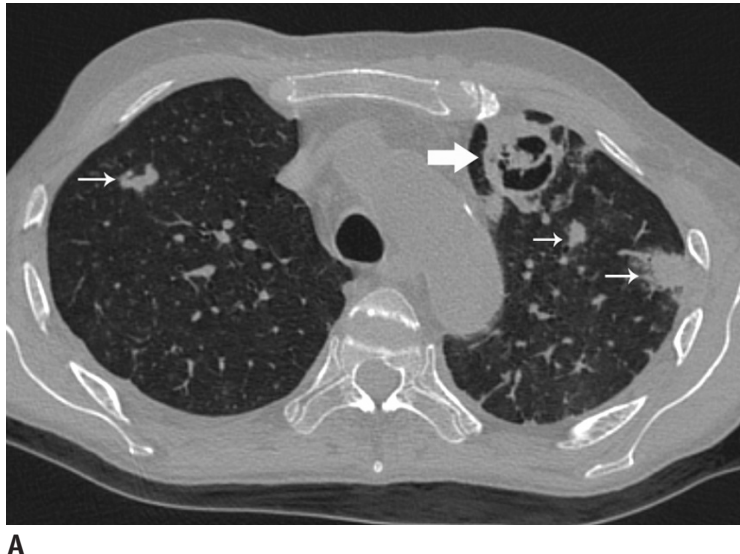

A

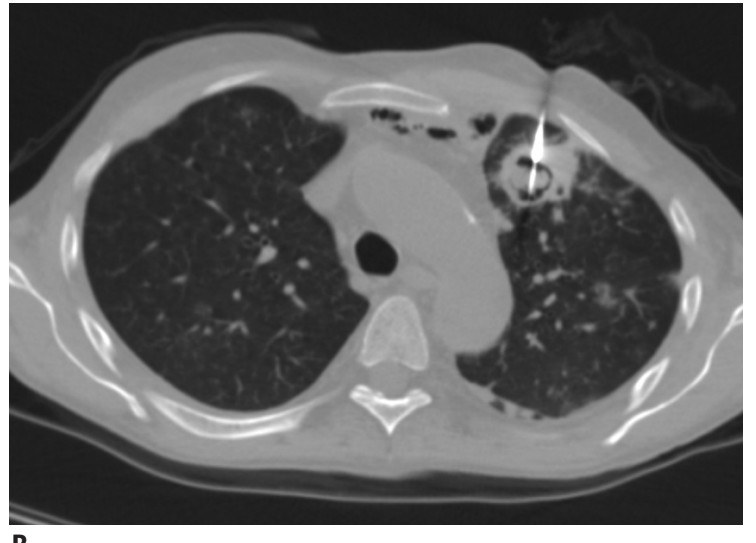

B

Fig. 27. Biopsy of cavitary lung lesion.

A. High resolution CT image of 42-year-old man who was on immunosuppressant medication following renal transplant. Cavitary lesion was seen in left lung with soft tissue ball inside (thick arrow) and multiple nodular lesions in bilateral lungs (thin arrows). B. Biopsy was taken from wall of cavity as well as intracavitary soft tissue ball. Biopsy results were suggestive of invasive aspergillosis. 
pneumothoraces were small and stable; and only less than $1 \%$ cases of pneumothorax required intervention. Whenever lung is punctured, the patients should be placed in a "biopsy side down" position for at least 1 hour immediately after completing the procedure and the patient should remain in the recumbent position for the next 4 hours. Although some of the authors have reported no benefits of putting patients in the "biopsy down position", this has been advocated by many others $(6,9,15,16)$. The rationale behind this maneuver is reducing the aeration of the punctured lung, resulting in the reduction of alveolar-to-pleural pressure gradient at the puncture site and accumulation of hemorrhagic fluid around needle path with an early sealing of the puncture tract $(8,15)$. No special post procedure positioning was required if lung was not transgressed. A blood patch technique has also been described to reduce chances of pneumothorax $(6,16)$. It required the injection of about 2-4 cc of patient's blood in the peripheral $2 \mathrm{~cm}$ of the needle tract, while removing guiding needle from the lung parenchyma.

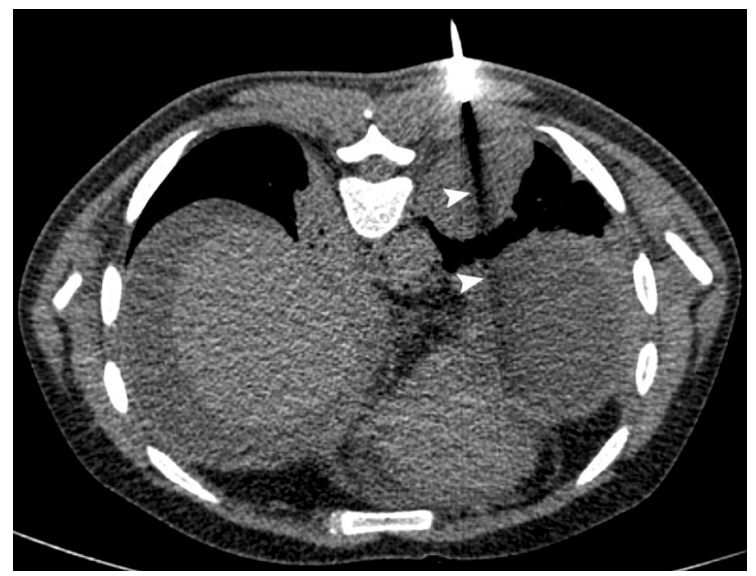

A

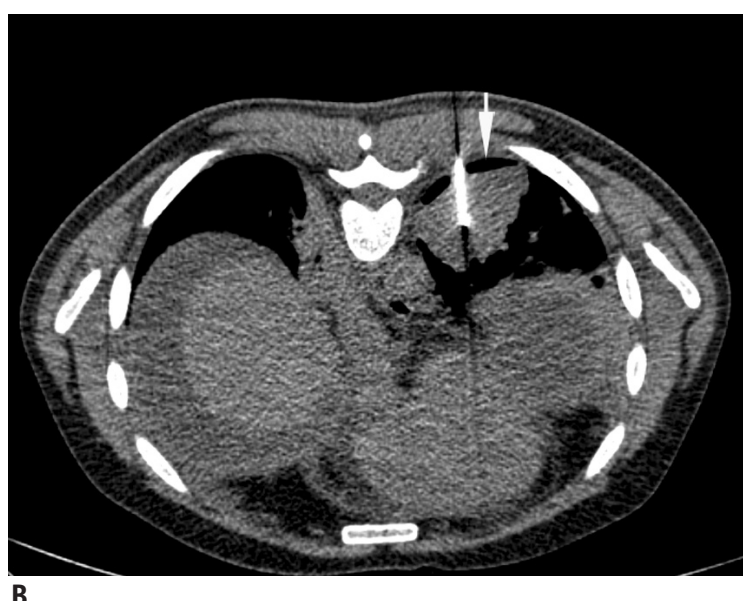

B

Fig. 28. Detection of early pneumothorax.

A. Prone CT scan of 32-year-old man with left lower lobe mass and peritoneal deposits. Guiding needle was positioned in chest wall and beam hardening artifact (arrowheads) showed needle trajectory. B. After needle was advanced into mass, air crescent (arrow) was noted separating lung mass from chest wall. This finding was suggestive of development of early pneumothorax and was confirmed on lung window images. Biopsy was quickly completed and patient was placed in "biopsy side down" position. Biopsy results revealed adenocarcinoma.

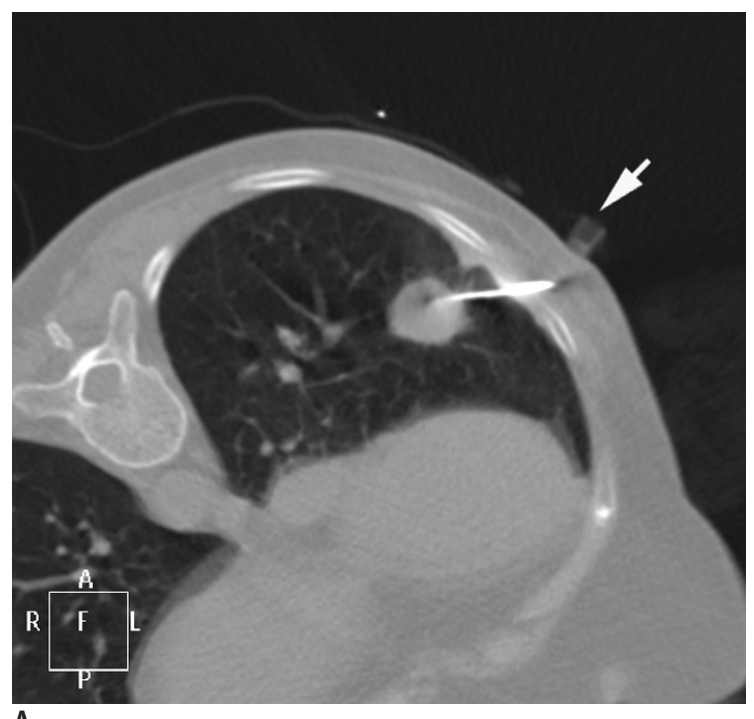

A

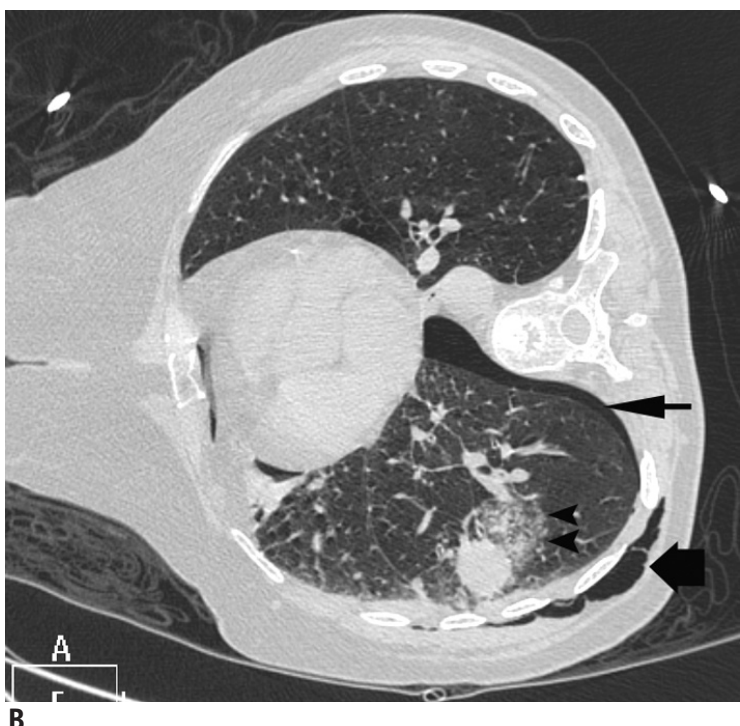

B

Fig. 29. Pneumothorax, surgical emphysema and alveolar hemorrhage following lung lesion biopsy.

A. Axial CT image of 73-year-old woman showed left lower lobe nodule. Plastic marker (arrow) was placed close to skin entry site. Due to excessive movement with respiration, lesion could be targeted after puncturing lung twice. B. Post biopsy image showed development of pneumothorax (thin arrow) and surgical emphysema of chest wall (thick arrow). Alveolar hemorrhage is also noted around lesion (arrowheads). Biopsy was suggestive of adenocarcinoma. 
Pulmonary hemorrhage may occur with or without hemoptysis. While hemorrhage is recorded in $5.0-16.9 \%$ cases, it manifests as hemoptysis in only $1.2-5.0 \%$ patients (2). If hemoptysis occurs, patients should be reassured and placed in the decubitus position with biopsy side down to prevent aspiration of blood in other areas of the lung (Fig. 29). In most of cases, hemoptysis is self limiting and settles down with conservative treatment; however, massive hemoptysis is the most dreaded complication of a lung biopsy and should be promptly treated. The risk of pulmonary hemorrhage increases in chronic inflammatory cavities due to the presence of hypertrophied bronchial arteries, vascular tumors, centrally located lesions, use of a cutting biopsy needle, pulmonary hypertension, and a deranged coagulation profile (6). In vascular lesions and in patients with bleeding disorders, small caliber needles should be used to reduce the risk of severe hemorrhage (6).

An air embolism is a very rare complication of PCNB, and it may affect the cerebral or coronary circulation (17). The probable mechanisms include either pulling of ambient air into the pulmonary vein via the biopsy needle, or by the formation of a broncho-venous fistula from the needle path or removed tissue core. Predisposing factors for air embolisms are coughing during the procedure, biopsy of consolidated lung, cavitary or cystic lesions, an associated vasculitis and use of a co-axial technique $(9,17)$. To prevent an air embolism the guiding needle should never be left without the inner stylet. During exchange of inner stylet with the biopsy needle, the hub of the guiding needle should be covered with the finger or thumb (7).

Significant chest wall hematoma and hemothorax are rare, but may develop if the intercostal or internal mammary

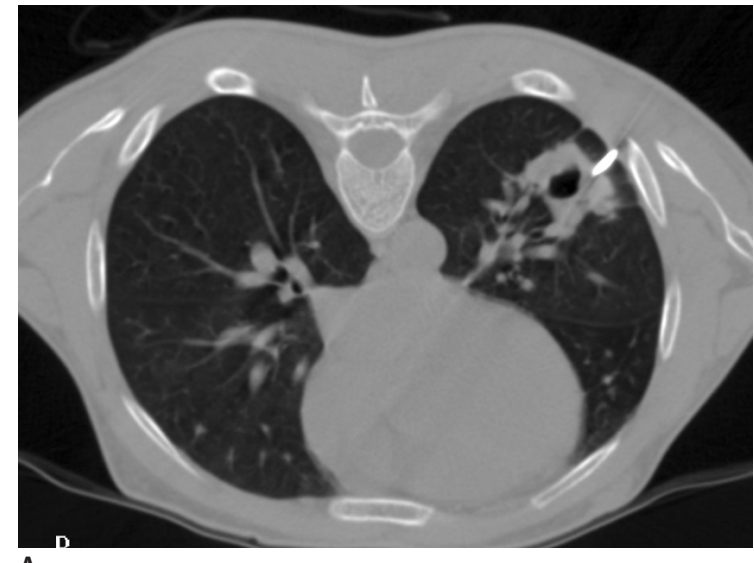

A

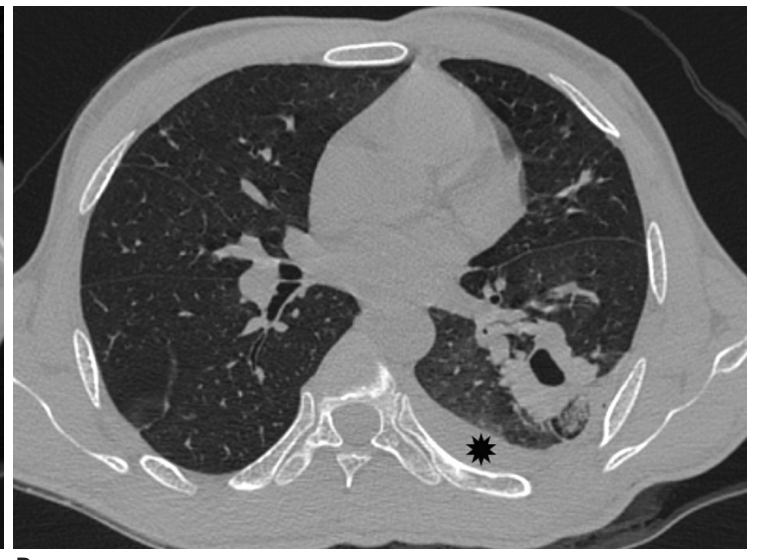

B

Fig. 30. Development of hemothorax following lung lesion biopsy.

A. Prone CT image of 41-year-old prison inmate who presented with hemoptysis and multiple cavitary lesions in both lungs. Guiding needle was placed at margin of lesion. B. Post biopsy supine scan showed development of hemothorax (star). Most likely, hemothorax developed due to intercostal artery injury as needle path was close to inferior rib margin.
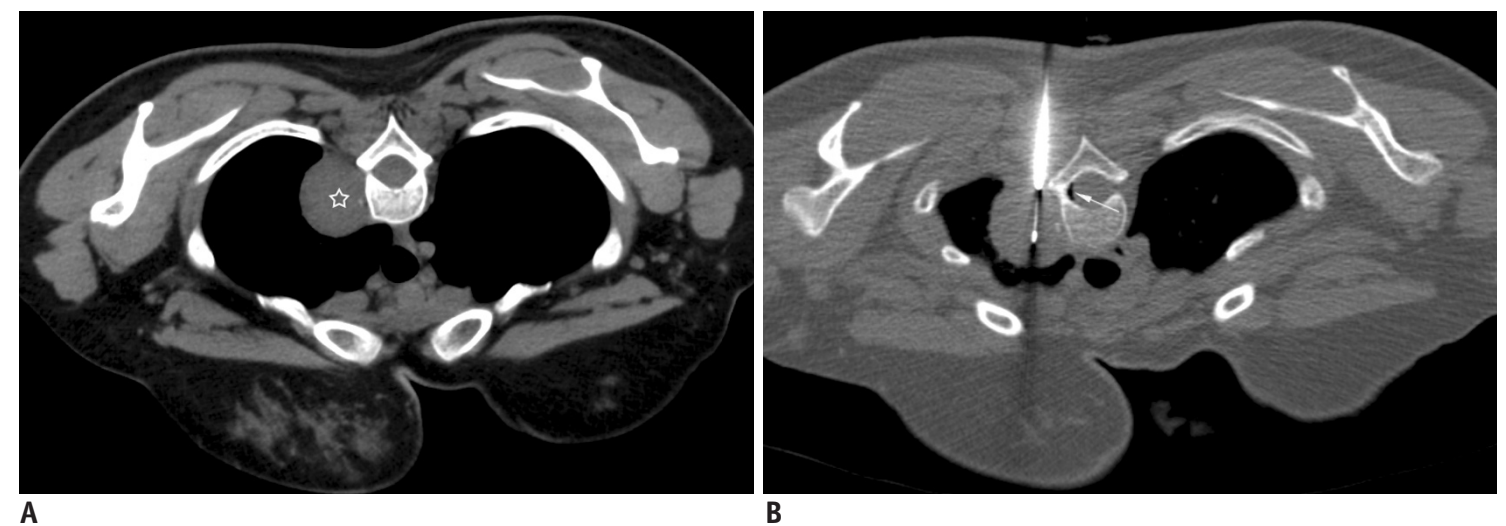

B

Fig. 31. Development of pneumorrhachis following biopsy of mediastinal lesion.

A. Localization CT image of 31-year-old woman showed well defined mass in right paravertebral location (star). B. Guiding needle was advanced till margin of lesion and biopsy needle is inserted into mass. Small crescent of air was noted in spinal canal (thin white arrow), which was suggestive of pneumorrhachis. Patient did not complain of any symptoms and was managed conservatively. Biopsy results were suggestive of schwannoma. 
arteries are injured during the biopsy procedure (Fig. 30). Pneumorrhachis, the presence of intraspinal air, has been rarely reported after anesthetic interventions (18). A single case of pneumorrhachis has been reported following chest tube insertion (18). We have observed this phenomenon in one patient, most likely due to the pulling of air through the nerve root sleeve and into the spinal canal (Fig. 31).

\section{SUMMARY}

In this pictorial review, we have presented various technical and anatomical aspects useful during biopsy of mediastinal and lung lesions. Familiarity of the operator with these methods will be helpful in obtaining adequate tissue material while minimizing complications.

\section{REFERENCES}

1. Klein JS, Salomon G, Stewart EA. Transthoracic needle biopsy with a coaxially placed 20 -gauge automated cutting needle: results in 122 patients. Radiology 1996;198:715-720

2. Manhire A, Charig M, Clelland C, Gleeson F, Miller R, Moss H, et al. Guidelines for radiologically guided lung biopsy. Thorax 2003;58:920-936

3. Cham MD, Henschke CI, Yankelevitz DF. Biopsy procedures of the lung, mediastnum, and chest wall. In: Kandarpa K, Machan L, eds. Handbook of interventional radiologic procedures, 4th ed. Philadelphia, USA: Wolters Kluwer/ Lippincott Williams \& Wilkins, 2011:478-485

4. Cham MD, Lane ME, Henschke CI, Yankelevitz DF. Lung biopsy: special techniques. Semin Respir Crit Care Med 2008;29:335349

5. Gupta S, Seaberg K, Wallace MJ, Madoff DC, Morello FA $\mathrm{Jr}$, Ahrar K, et al. Imaging-guided percutaneous biopsy of mediastinal lesions: different approaches and anatomic considerations. Radiographics 2005;25:763-788

6. Moore EH. Technical aspects of needle aspiration lung biopsy: a personal perspective. Radiology 1998;208:303-318
7. Tsai IC, Tsai WL, Chen MC, Chang GC, Tzeng WS, Chan SW, et al. CT-guided core biopsy of lung lesions: a primer. $\mathrm{Am} \mathrm{J}$ Roentgenol 2009;193:1228-1235

8. Kinoshita F, Kato T, Sugiura K, Nishimura M, Kinoshita T, Hashimoto $M$, et al. CT-guided transthoracic needle biopsy using a puncture site-down positioning technique. $\mathrm{Am} \mathrm{J}$ Roentgenol 2006;187:926-932

9. Wallace $A B$, Suh RD. Percutaneous transthoracic nnedle biopsy:special considerations and techniques used in lung transplant recipients. Semin Intervent Radiol 2004;21:247-258

10. Cox JE, Chiles C, McManus CM, Aquino SL, Choplin RH. Transthoracic needle aspiration biopsy: variables that affect risk of pneumothorax. Radiology 1999;212:165-168

11. Yamagami T, Kato T, Iida S, Hirota T, Nishimura T. Percutaneous needle biopsy for small lung nodules beneath the rib under CT scan fluoroscopic guidance with gantry tilt. Chest 2004; 126:744-747

12. Paulson EK, Sheafor DH, Enterline DS, McAdams HP, Yoshizumi TT. CT fluoroscopy-guided interventional procedures: techniques and radiation dose to radiologists. Radiology 2001;220:161-167

13. Kim GR, Hur J, Lee SM, Lee HJ, Hong YJ, Nam JE, et al. CT fluoroscopy-guided lung biopsy versus conventional CT-guided lung biopsy: a prospective controlled study to assess radiation doses and diagnostic performance. Eur Radiol 2011;21:232239

14. Bandoh S, Fujita J, Fukunaga Y, Yokota K, Ueda Y, Okada H, et al. Cavitary lung cancer with an aspergilloma-like shadow. Lung Cancer 1999;26:195-198

15. Collings CL, Westcott JL, Banson NL, Lange RC. Pneumothorax and dependent versus nondependent patient position after needle biopsy of the lung. Radiology 1999;210:59-64

16. Wu CC, Maher MM, Shepard JA. Complications of CT-guided percutaneous needle biopsy of the chest: prevention and management. AJR 2011;196:W678-W682

17. Hare SS, Gupta A, Goncalves AT, Souza CA, Matzinger F, Seely JM. Systemic arterial air embolism after percutaneous lung biopsy. Clin Radiol 2011;66:589-596

18. Oertel MF, Korinth MC, Reinges MH, Krings T, Terbeck S, Gilsbach JM. Pathogenesis, diagnosis and management of pneumorrhachis. Eur Spine J 2006;15 Suppl 5:S636-S643 